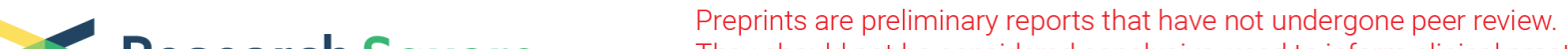

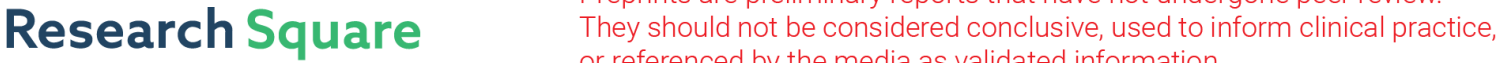 or referenced by the media as validated information.
}

\section{MiR-21-5p regulates extracellular matrix degradation and angiogenesis in TMJOA by targeting Spry1.}

Shixing Ma

Shandong University

Aobo Zhang

Shandong University

Xiaole Li

Shandong University

Shizhou Zhang

Shandong University

Shaopeng Liu

Shandong University

Haoming Zhao

Shandong University

Shichao Wu

Liaocheng People's Hospital

Lei Chen

Shandong University

Chuan Ma

Shandong University

Huaqiang Zhao (D MsxZab@163.com )

College of stomatology, Shandong University https://orcid.org/0000-0002-9632-1962

Research article

Keywords: miR-21-5p, Spry1, TMJOA, VEGF, MMP-13, ERK-MAPK signalling pathway

Posted Date: December 20th, 2019

DOl: https://doi.org/10.21203/rs.2.19356/v1

License: (c) (i) This work is licensed under a Creative Commons Attribution 4.0 International License.

Read Full License 


\section{Abstract}

Background: Due to the lack of research on the pathological mechanism of temporomandibular joint osteoarthritis (TMJOA), there are few effective treatment measures in the clinic. In recent years, microRNAs (miRs) have been demonstrated to play an important role in the pathogenesis of osteoarthritis $(\mathrm{OA})$ by regulating a variety of target genes, and the latest evidence shows that miR-21-5p is specifically overexpressed in OA. The purpose of this project was to clarify whether miR-21-5p can regulate the TMJOA process by targeting Spry1.

Methods: TMJOA was induced by a unilateral anterior crossbite (UAC) model, and the effect of miR-21-5p knockout on TMJOA was evaluated by toluidine blue and immunohistochemical staining. Primary mouse condylar chondrocytes (MCCs) were isolated, cultured and transfected with a series of mimics, inhibitors, siRNA-Spry1 or cDNA Spry1. Western blotting, RT-qPCR, immunofluorescence staining, and toluidine blue staining were used to detect the effect of miR-21-5p and its target gene Spry 1 on the expression of MMP13, VEGF and p-ERK1/2 in TMJOA. The effect of miR-21-5p on angiogenesis was evaluated by chick embryo CAM assay and Western blotting.

Results: In the UAC model, the cartilage thickness and extracellular matrix of miR-21-5p knockout mice were less damaged. Luciferase experiments confirmed that Spry 1 was the direct target of miR-21-5p. The expression levels of Spry1, MMP-13, VEGF and p-ERK1/2 in MCCs transfected with miR-21-5p mimic were higher than those in the inhibitor group. Under the simulated inflammatory environment of IL-1 $\beta$, the expression levels of MMP-13, VEGF and p-ERK1/2 were positively correlated with miR-21-5p, while Spry 1 was negatively correlated with miR-21-5p. Inhibition of miR-21-5p expression and overexpression of Spry1 enhanced the inhibition of MMP-13, VEGF and p-ERK1/2 expression. MiR-21-5p had a significant role in promoting angiogenesis in the chick embryo CAM assay, and this role was clearly mediated by the ERK-MAPK signalling pathway.

Conclusion: This study verified that miR-21-5p can promote the process of TMJOA by targeting Spry1, which provides a new direction for future research on the treatment of this disease.

\section{Background}

Temporomandibular disorders (TMD) are a complex and controversial disease [1]. Temporomandibular joint osteoarthritis (TMJOA) is characterized by destruction and loss of the articular cartilage matrix, subchondral osteosclerosis, synovitis and osteophyte, which is one of the most serious diseases in the TMD classification [2]. The temporomandibular joint (TMJ) is the only joint composed of fibrocartilage, so it is different from other joints composed of hyaline cartilage in structure and function. Degenerative changes in articular cartilage in patients with TMJOA are generally considered to be due to selfreconstruction after dysfunction of the joint structure. The direct cause of degenerative changes is likely to be a decrease in cartilage surface adaptability or a joint overload. However, it is still difficult to explain the pathophysiological mechanism that leads to the progression of this disease. Further studies of 
extracellular regulatory factors and intracellular signalling mechanisms that regulate articular cartilage homeostasis have helped to discover new therapeutic targets for osteoarthritis [3].

Due to their specific anatomy and location, chondrocytes survive in an environment of lack of blood vessels and hypoxia and maintain tissue homeostasis. Blood vessels originating in the subchondral bone can enter the cartilage microenvironment which lacks blood vessels through further growth and breaking through the tide mark, thus stimulating the ossification of articular cartilage and leading to OA. In the subchondral bone of $\mathrm{OA}$, the severity of structural changes is positively correlated with the expression of vascular endothelial growth factor (VEGF) secreted by chondrocytes [4]. In addition, new research shows that VEGF can play a key role in inducing chondrocyte apoptosis and cartilage degeneration of the TMJ by increasing the expression of matrix metalloproteinases (MMPs) [5]. MMPs are a family of major enzymes that degrade extracellular collagen and aggrecan (ACAN) in OA. In chondrocytes, VEGF can promote the secretion of MMP-1, MMP-3 and especially MMP-13 [6, 7]. In addition, the activation of the ERK-MAPK signalling pathway has been confirmed to promote the secretion of MMPs in chondrocytes and osteoblasts, thus promoting the development of OA [8]. Therefore, abnormal expression of VEGF and activation of the ERK-MAPK signalling pathway are important factors leading to angiogenesis and extracellular matrix degradation of TMJOA.

MicroRNAs are a class of endogenous non-coding RNAs 19-25 nucleotides in length that can regulate the expression of target genes through complementary pairing with the target gene 3\- untranslated region (3区UTR), resulting in mRNA degradation or translational inhibition [9]. Recent studies have confirmed that microRNAs can participate in cartilage formation, cartilage degradation and OA development by regulating various cell processes, such as apoptosis, cell differentiation, proliferation, and matrix remodelling $[10,11,12]$. Thus, microRNAs play an important role in the pathogenesis of $O A$. Through the use of Gene Expression Omnibus (GEO) analysis and the detection of miR-21-5p expression in $O A$ patient cartilage, it has been found that the expression of miR-21-5p in $O A$ is significantly upregulated $[13,14]$. In addition, the latest study found that miR-21-5p can promote the degradation of extracellular matrix by regulating its target gene fibroblast growth factor 18 (FGF18) and suggested that miR-21-5p may be a new target for OA treatment [14]. It can be considered that miR-21-5p, as a new target closely related to $\mathrm{OA}$, is likely to play a crucial role in the pathogenesis and progression of TMJOA.

Sproutys (Sprys) are negative regulators of ERK1/2 activation induced by RTK $[15,16]$, and Spry 1 is one of the four identified mammalian Spry homologs (Spry1, 2, 3, 4) [17]. Studies have shown that the main function of Spry 1 is to inhibit the Ras/Raf/ERK signalling pathway, and the absence of Spry 1 activates phosphorylation of the ERK-MAPK signalling pathway $[15,18]$. The protein encoded by the Spry 1 gene also exhibits this inhibitory function under the stimulation of a series of growth factors, and VEGF is one of the major stimulators of the Spry1 protein [16]. It can be seen from the above research background that the abnormal expression of VEGF and MMPs and activation of the ERK-MAPK signalling pathway play a critical role in the pathogenesis of TMJOA, so Spry1 is likely to participate in the inflammatory destruction process of TMJOA. Interestingly, we found that Spry 1 was the direct target of miR-21-5p by bioinformatics analysis and dual luciferase assay. Therefore, we hypothesized that miR-21-5p could 
upregulate the secretion of VEGF and MMPs and activate the ERK-MAPK signalling pathway by targeting Spry 1 to promote the process of TMJOA.

This experiment was the first to investigate the effects of miR-21-5p and its target gene Spry 1 on the degradation and angiogenesis of TMJ. We found that miR-21-5p knockout (KO) mice had a lower degree of TMJ cartilage destruction than the wild type (WT) mice in the established TMJOA model. In vitro results showed that miR-21-5p could inhibit the expression of Spry1, resulting in the upregulation of MMP-13, VEGF expression and phosphorylation of ERK-MAPK signalling pathway components.

\section{Methods}

Animals and dental operation

All procedures and protocols were approved by the Ethics Committee of Shandong University. Twenty WT male C57BL/6N mice (6-8 weeks of age, weight 18-21 g) were obtained from the Animal Centre of

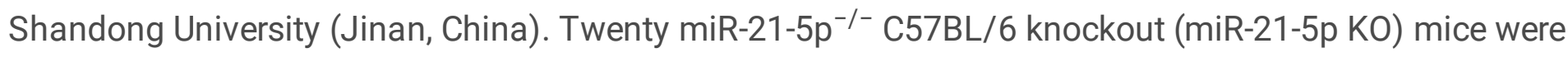
bred from miR-21-5p KO mice (two males, two females) purchased from Southeast University (Nanjing, China). KO mouse genotyping results are listed in Supplementary 1.WT mice were randomly divided into a control group (WT-NC) and unilateral anterior crossbite group (WT-UAC). MiR-21-5p KO mice were randomly divided into a control group (KO-NC) and unilateral anterior crossbite group (KO-UAC). Metal tubes were placed in the left maxillary incisor and mandibular incisor of the intervention group, and the intervention time was 3 weeks. The operation steps of the UAC model were performed as described [19]. Mice in the NC groups underwent all procedures, but no metal tube was bonded. All mice received a standardized diet.

Histology and immunohistochemistry staining (IHC)

After three weeks, six mice (12 TMJs) were randomly selected from each group for dissection. After fixation in paraformaldehyde, the samples were decalcified in EDTA for four weeks, then embedded in paraffin, and $5 \mu \mathrm{m}$ thick sections were prepared. After deparaffinization, the sections were stained with toluidine blue (TB) (Sigma-Aldrich, Poole, UK). Collagen fibres and ACAN are the main extracellular matrix components of cartilage and can be stained blue-violet by the basic dye TB. Image-Pro Plus 6.0 software was used to quantify the average optical density (AOD) values of the stained area. Blue-stained areas were selected as the uniform standard for evaluating all images. Each image was analysed to obtain the integrated optical density (IOD) and the area of the pixel (AREA) of the tissue. Finally, the AOD (AOD = IOD/AREA) was determined. Using the image scale $(50 \mu \mathrm{m})$ as the standard, the cartilage thickness (mm) at 5 positions was measured for each image, and the average value was obtained.

For $\mathrm{IHC}$, the sections were dewaxed and $0.1 \%$ trypsin was applied to each section for 10 min at $37^{\circ} \mathrm{C}$, and the endogenous peroxidase activity of the sections was quenched using $3 \% \mathrm{H}_{2} \mathrm{O}_{2}$ for $10 \mathrm{~min}$. Then, sections were blocked with $5 \%$ bovine serum albumin (BSA) for 30 min and incubated with primary antibody against aggrecan (ACAN) $(1: 100 ; \mathrm{Abcam}, \mathrm{MA}, \mathrm{USA})$ at $4{ }^{\circ} \mathrm{C}$ overnight. Then, the sections were 
incubated with the secondary antibody and horseradish peroxidase-conjugated avidin (BOSTER, SA1022, China). For IHC staining, the percentages of positively stained cells were determined. The AOD of the positive area was measured.

Target prediction and luciferase reporter assay

To predict the target relationship between miR-21-5p and Spry1, a miRNA prediction software packages, miRanda (http://www.microrna.org/microrna/home.do), which is widely used to confirm the theoretical target genes of miRNAs, was used in this study. To further verify the reliability of the target gene prediction, MCCs were cotransfected with a luciferase reporter containing the target fragment (Spry1-3' UTR-WT) or with a vector carrying the mutant-type 3' UTR of Spry1 (Spry1-3' UTR-MUT) and with miR-21$5 p$ mimic or the corresponding negative control (NC). The amplified primers sequences of 3'UTR of Spry1 were as follows: Spry1 sense: CTT TGT GCC TAC CCT GCT TGC TCT GCT ACC; Spry1 anti-sense: AGG GCG GTG GGT CCA GTC GTA ACA GC. After $48 \mathrm{~h}$, the luciferase activity was calculated using the dualluciferase reporter assay system according to the manufacturer's instructions (Promega, Madison, WI, USA).

Culture and identification of condylar chondrocytes

MCCs were isolated from mice (2-week-old, male, $5 \mathrm{~g}$ ). Samples were minced into pieces of less than 1 $\mathrm{mm}^{3}$ with microsurgical scissors, followed by digestion at $37^{\circ} \mathrm{C}$ with $3 \mathrm{mg} / \mathrm{ml}$ dispase (Sigma-Aldrich, USA) $1 \mathrm{x}$ and $2.5 \mathrm{mg} / \mathrm{ml}$ collagenase type II in DMEM for $2 \mathrm{~h}$ with stirring every $20 \mathrm{~min}$. The single cell suspensions were cultured $\left(5 \% \mathrm{CO}_{2}, 37^{\circ} \mathrm{C}\right)$ in $79 \%$ DMEM supplemented with $20 \% \mathrm{FBS}, 100 \mathrm{mg} / \mathrm{ml}$ streptomycin and $100 \mathrm{mg} / \mathrm{ml}$ penicillin solution for 5 to 7 days before use. The cells were digested with trypsin-EDTA and passaged into a T25 culture flask for subsequent experiments. Additionally, the chondrocytes were validated through immunocytochemical identification of type II collagen (Supplementary 2).

Cell transfection

MCCs were cultured to $85 \%$ confluence in 6-well plates. Transfection of MCCs using Lipofectamine 2000 (Thermo Fisher, USA, catalogue no 11668-019, $4 \mu \mathrm{l} /$ well) was performed with the synthetic precursors of miR-21-5p called miR-21-5p mimic, mimic negative control (mimic NC), miR-21-5p inhibitor and inhibitor negative control (inhibitor NC) (Genepharma, Shanghai, China). The cells were transfected with miR-21-5p mimic or inhibitor to a final concentration of $50 \mathrm{nM}$ with Lipofectamine 2000 according to the manufacturer's protocols. After $6 \mathrm{~h}$, the medium was replaced with DMEM supplemented with $20 \% \mathrm{FBS}$. After $48 \mathrm{~h}, \mathrm{RNA}$ and protein were extracted and analysed.

The vector for Spry1 overexpression was GV141, the enzyme digestion cloning site was Xhol/Kpnl, and the empty vector was used as the control (GeneChem, Shanghai, China). The target gene sequence for chemical synthesis and the primer sequence for identification of the recombinant plasmid is listed in Supplementary 3. MCCs were transfected with the aforementioned vectors using Lipofectamine 2000. For 
Spry1 knockdown, chondrocytes were transfected with small interference RNA of mouse Spry1 (si-Spry1) to suppress Spry1 expression. Chondrocytes were transfected with Spry1-specific small interfering RNA (si-Spry1) or negative control siRNA (si-NC) (GeneChem, Shanghai, China) using Lipofectamine 2000. After transfection, the chondrocytes were incubated with $10 \mathrm{ng} / \mathrm{ml} \mathrm{IL-1 \beta}$ (R\&D systems, Abingdon, UK) for another $24 \mathrm{~h}$. The transfection efficiency was determined using Western blotting. The sequences of the mimic, inhibitor, siRNA are listed in Supplementary Table 1.

\section{Western blotting}

Condylar cartilage was incubated in liquid nitrogen and ground to a fine powder. MCCs were collected from plates and washed with DPBS. Tissue and cells were lysed using RIPA with $1 \%$ phenylmethanesulfonyl fluoride (PMSF) (Beyotime, China) followed by centrifugation at 12,000 rpm for $15 \mathrm{~min}$ at $4{ }^{\circ} \mathrm{C}$, and the resulting supernatants were quantified by the bicinchoninic acid (BCA) assay. A $10 \%$ sodium dodecyl sulfate separation gel and a concentration gel were prepared. Transfer of the proteins to nitrocellulose membranes was carried out at $60 \mathrm{~V}$ for $1 \mathrm{~h}$ and $120 \mathrm{~V}$ for $0.5 \mathrm{~h}$. The polyvinylidene difluoride membranes (Millipore, Bedford, MA, United States) were blocked for $2 \mathrm{~h}$ with $5 \%$ non-fat milk. The membrane was then incubated with primary antibodies for $12 \mathrm{~h}$. The blots were washed three times and incubated with secondary antibodies. After washing, the membranes were developed using an ECL Western blotting kit (Beyotime, Shanghai China). Finally, the blots were analysed quantitatively. The following antibodies were used: rabbit anti-Spry1 (1:1000; Abcam, MA, USA), rabbit anti-MMP13 (1:1000; Abcam, MA, USA), rabbit anti-VEGF (1:1000; Abcam, MA, USA), rabbit anti-ACAN (1:500; Abcam, MA, USA), rabbit anti-ERK ( 1:1000; Cell Signaling Technology, USA), rabbit anti-phosphoERK (1:1000; Cell Signaling Technology, USA), rabbit anti- $\beta$-actin (1:1000; Beyotime, China), rabbit anti$\lg \mathrm{g}(1: 5,000 ;$ Beyotime, China).

Measurement of miRNAs and mRNA expression

Total RNA was extracted from the tissues and MCCs using TRIzol Reagent (Invitrogen). For quantitative detection of miRNA, a TaqMan miRNA assay kit (Thermo Fisher, USA) was used. Purified miRNA was reverse transcribed using miRNA-specific stem-loop RT primers (Applied Biosystems, USA). Following the manufacturer's instructions, reverse transcription-quantitative PCR (RT-qPCR) was performed in a 7500 Real Time PCR system (Applied Biosystems, USA) using SYBR® Premix Ex Taq II Kit (TaKaRa, Japan). Gene expression was normalized to U6 small nuclear RNA expression. The relative gene expression was measured by using the comparative threshold cycle $\left(2^{\wedge}-\Delta \Delta C t\right)$ method, and $\beta$-actin served as an internal control. The reaction mixtures were incubated at $95^{\circ} \mathrm{C}$ for $10 \mathrm{~min}$, followed by 40 cycles of $20 \mathrm{~s}$ at $95^{\circ} \mathrm{C}$ and $60 \mathrm{~s}$ at $55^{\circ} \mathrm{C}$. The primers used are shown in Supplementary 4 .

Toluidine blue staining

After treatment according to the experimental design, MCCs were washed three times with DPBS before staining, fixed in $4 \%$ buffered paraformaldehyde for at least $20 \mathrm{~min}$ at room temperature and washed 
with DPBS. Cells were then stained in toluidine blue solution for 10 min at $37^{\circ} \mathrm{C}$ and washed with DPBS for 3 min. The staining results were observed by microscopy and quantified.

Cell immunofluorescence

MCCs were washed with DPBS three times and fixed with 4\% paraformaldehyde for 15 min and then rinsed in DPBS three times, 5 min each time. For Spry 1 and ACAN detection, the cells were incubated with rabbit anti-Spry1 (1:100; Abcam, MA, USA) and rabbit anti-ACAN (1:50; Abcam, MA, USA) at $4{ }^{\circ} \mathrm{C}$, overnight. FITC-labelled goat rabbit anti-lgG (Beyotime, Shanghai, China) was used as a secondary antibody and incubated for $1 \mathrm{~h}$ at $37^{\circ} \mathrm{C}$. The chondrocyte nuclei were restained with 4', 6-diamidino-2phenylindole (DAPI) (Solarbio, Beijing, China) for $10 \mathrm{~min}$. The analysis of fluorescence was performed with a confocal scanning microscope system (Carl Zeiss, Nikon).

Chick embryo in vivo angiogenesis assay

SPF-grade white eggs were purchased from Merial-Vital Company (Beijing, China). For the vascularization experiment, the cells were divided into four groups: NC, miR-21-5p inhibitor, miR-21-5p mimic, miR-21-5p mimic + U0126. After 2 days of transfection, the cells of each group were resuspended in serum-free medium (15 $\mu$ l), mixed with BD Matrigel ${ }^{\mathrm{TM}}$ Basement Membrane Matrix (Corning, USA) of equal volume (15 $\mu \mathrm{l}$ each), and the mix was implanted into each egg, after which the eggs were returned to the incubator for further incubation. The embryo were incubated at $37.5 \pm 0.5^{\circ} \mathrm{C}$ and relative humidity of $60-$ $80 \%$. The position of the air chamber was marked on the surface of normal egg shells, part of the egg shell was peeled off to expose the egg white membrane and the intima was carefully removed with sterile tweezers. At embryonic day 10, Matrigel mixed with different groups of cells was implanted into each egg. After $72 \mathrm{~h}$, images of the region surrounding the Matrigel were taken, and vascular branches were calculated.

\section{Statistical analysis}

Bars represent the standard error of the mean (SEM) from three independent experiments. SPSS 20.0 and Image-Pro Plus 6.0 software were used for statistical and image analyses. The data were graphically presented using GraphPad Prism 7.0. Statistical significance between two groups was assessed by Student's t-test. For multiple comparisons, one-way analysis of variance (ANOVA) followed by NewmanKeuls post hoc tests was used. Data with a p-value less than or equal to 0.05 were considered significant.

\section{Result}

Knockout of miR-21-5p reduces surgically induced TMJOA progression

Statistical analysis of Image-Pro Plus 6.0 software results showed that the AOD in the KO-UAC group was higher than that in the WT-UAC group (Fig. 1a, 1c). Furthermore, the results of the thicknesses of cartilage layers showed thinner layers in the WT-UAC group than in the KO-UAC group (Fig. 1a, 1b). There was no 
statistically significant difference between the WT-NC group and the KO-NC group in the AOD values and thickness of cartilage layers (Fig. 1a, 1b). In addition, we detected ACAN protein by immunohistochemistry $(\mathrm{IHC})$ and determined that ACAN expression was significantly lower in the UAC group than in the NC group, and there was no significant difference between the WT-NC group and the KONC group; however, the expression of ACAN was higher in the KO-UAC group than in the WT-UAC group (Fig. 1d, 1e). These data suggest a chondroprotective effect of miR-21-5p KO on osteoarthritis mice and inhibition of cartilage matrix loss in UAC-induced OA.

Spry1 was a target gene of miR-21-5p

We first predicted that Spry1 was a target gene of miR-21-5p through analysis of a miRNA target databases and identified the potential binding sequence of the 3' UTR of Spry1 and miR-21-5p (Fig. 2a). To further confirm this result, we performed a dual luciferase reporter assay. MCCs were transfected with miR-21-5p mimic and the corresponding negative control (NC) and then co-transfected with Spry1-3' UTRWT or Spry1-3' UTR-MUT. Luciferase experiments showed that the Spry1-3' UTR-WT group had a significant decrease in luciferase activity, but the Spry1-3' UTR-MUT group had no significant change in luciferase activity (Fig. 2b). This confirmed that Spry1 is a downstream target of miR-21-5p in chondrocytes. In addition, we found that Spry 1 protein expression was significantly downregulated after transfection of MCCs with miR-21-5p mimic, while Spry1 protein expression was significantly upregulated after transfection of miR-21-5p inhibitor (Fig. 3d, 3f). These results support the hypothesis that Spry1 is a direct target of miR-21 in chondrocytes.

The effect of miR-21-5p overexpression or silencing on the chondrocyte phenotype

MiR-21-5p mimic and inhibitor were transfected into MCCs to verify the role of miR-21-5p in MCCs and to detect changes in the mRNA and protein expression of Spry1, VEGF, MMP-13, ACAN and ADAMTS5. The RT-qPCR results showed that the expression of miR-21-5p was significantly increased in MCCs transfected with miR-21-5p mimic compared with the mimic NC group, while miR-21-5p was significantly inhibited in the miR-21-5p inhibitor group (Fig. 2c). The mRNA and protein expression levels of Spry1, VEGF and MMP-13 were significantly increased in the miR-21-5p mimic group compared with the mimic NC group, while the expression level of ACAN was downregulated (Fig. 2d, 2e). In contrast, the mRNA and protein expression of Spry1, VEGF and MMP-13 were decreased, and the expression of ACAN was upregulated in the miR-21-5p inhibitor group compared with the inhibitor NC group (Fig. 2f, 2 g).

IL-1 $\beta$-induced degradation of the extracellular matrix of condylar cartilage is regulated by miR-21-5p

IL-1 $\beta$ was used to induce an inflammatory state in MCCs. As shown in Fig. 4, the expression level of miR21-5p was significantly increased in IL-1 $\beta$-treated MCCs (Fig. 3a). The protein expression of the corresponding target gene Spry 1 was inhibited, and the expression levels of VEGF, MMP-13 and p-ERK1/2 were significantly increased (Fig. 3b). To demonstrate that miR-21-5p can promote the degradation of extracellular matrix under the inflammatory induction of IL-1 3 , we transfected MCCs with miR-21-5p mimic or miR-21-5p inhibitor, and the corresponding group was treated with IL-1 $\beta$ for $24 \mathrm{~h}$ after 
transfection. Under treatment with IL-1 $\beta$, the expression of VEGF, MMP-13 and p-ERK1/2 in the miR-21-5p mimic group was significantly upregulated (Fig. 3c), while the upregulation of VEGF, MMP-13 and pERK1/2 was significantly attenuated in the miR-21-5p inhibitor group (Fig. 3d). In addition, we evaluated the change in matrix content by performing toluidine blue staining in MCCs. The results showed that the average optical density values of the IL-1 $\beta$-treated group were lower than those of the untreated group. Compared with the negative control, overexpression of miR-21-5p reduced matrix staining, while silencing miR-21-5p increased matrix staining, especially in the presence of IL-1ß-induced MCCs (Fig. 4a, 4b). These results suggest that miR-21-5p promotes IL-1 $\beta$-mediated extracellular matrix catabolism.

IL-1 $\beta$ induces degradation of the extracellular matrix of MCCs and can be regulated by miR-21-5p targeting Spry 1

We transfected MCCs with miR-21-5p mimic or inhibitor, and then treated the cells with IL-1 $\beta$ according to the experimental groups. After that, Spry1 and ACAN were stained by immunofluorescence (Fig. 5). Compared with that in the NC group, the fluorescence intensity of Spry 1 and ACAN in the miR-21-5p mimic group, IL-1 $\beta+N C$ group and IL-1 $\beta+$ mimic group was significantly decreased. In addition, the fluorescence intensity of Spry 1 and ACAN in the IL-1 $\beta+$ inhibitor group was significantly higher than that in the IL-1 $\beta+$ NC group and the IL-1 $\beta+$ mimic group. More importantly, the fluorescence intensity of Spry 1 and ACAN in the IL-1 $\beta+$ mimic group was significantly lower than that in the miR-21-5p mimic group and the IL-1 $\beta+$ NC group. Therefore, we believe that the process by which miR-21-5p promotes the degradation of the MCC extracellular matrix is likely to be related to its targeted regulation of Spry 1 expression.

To further demonstrate that miR-21-5p promotes extracellular matrix degradation under an inflammatory state through regulation of its target gene Spry1, we used small interfering RNA (si-Spry1) and a Spry1 ectopic expression plasmid (cDNA Spry1) to transfect MCCs. The knockdown of Spry1 expression induced by IL-1 $\beta$ significantly increased the expression of VEGF and MMP-13 and promoted the activation of the ERK/MAPK signalling pathway (Fig. 6a). This result is consistent with the trend observed with miR-21-5p mimic (Fig. 4a, 4c). Transfection of ectopic expression plasmids showed that the expression of VEGF, MMP-13 and p-ERK1/2 in the IL-1 $\beta+$ CDNA Spry 1 group was significantly downregulated compared with that in the IL-1 $\beta+$ CDNA NC group (Fig. 6b). In addition, we also cotransfected miR-21-5p inhibitor with Spry1 small interfering RNA and ectopic expression plasmid and showed that overexpression of Spry 1 could enhance the inhibitory effect of miR-21-5p inhibitor on VEGF, MMP-13 and p-ERK1/2 protein expression levels in the inflammatory state (Fig. 7b). Interfering with the expression of Spry1 attenuated the inhibition (Fig. 7a). This indicates that miR-21-5p affects the degradation of extracellular matrix induced by IL-1 $\beta$ via the targeted regulation of Spry1.

MiR-21-5p promotes angiogenesis in MCCs that is mediated by the ERK/MAPK signalling pathway

To detect the effect of miR-21-5p on the angiogenesis of TMJ cartilage and to verify whether the ERKMAPK signalling pathway is involved in the process of miR-21-5p-induced angiogenesis, we transfected MCCs with miR-21-5p mimic or inhibitor, and the ERK-MAPK inhibitor U0126 was used in MCCs 
transfected with miR-21-5p mimic. The number of blood vessels in each group of eight chicken embryos was measured. Compared with that in the NC group, the number of vascular branches in the miR-21-5p mimic group increased significantly, while that in the miR-21-5p inhibitor group was the smallest. In addition, the number of new vessels in the miR-21-5p mimic and U0126 co-intervention group was significantly lower than that in the miR-21-5p mimic group but was closer to that in the NC group (Fig. 8a, 8b)(Table 2). To verify that U0126 can significantly inhibit the activation of $p$-ERK1/2, the expression of $p$ ERK protein was detected (Fig. 8c). Then, the expression of VEGF in the mimic + U0126 group was significantly lower than that in the mimic group (Fig. 8d), which indicated that the activation of the ERKMAPK signalling pathway in MCC is likely to regulate angiogenesis by promoting VEGF expression. Based on the above results, we believe that the expression of miR-21-5p in MCCs promotes the angiogenic activity of endothelial cells, which is at least partially mediated by the ERK-MAPK signalling pathway.

\section{Discussion}

At present, researches on the mechanism of microRNA regulation of OA disease development mainly focuses on large joints, such as knee, hip and finger joints, and there is little research on the mechanism of TMJOA. Our experiments aimed to investigate the possible mechanism of action of miR-21-5p in condylar cartilage inflammatory degeneration by establishing a UAC mouse model to induce cartilage structural dysfunction and using IL-1 $\beta$ to induce the inflammatory state of MCCs.

In recent years, the UAC model has been widely used to induce TMJOA, that is, to change the occlusal load distribution and induce $O A$ through experimental unilateral anterior cross occlusion, leading to the loss of subchondral bone in the TMJ [20,21,22]. In vitro experiments have used the inflammationinducing factor IL-1 $\beta$, which is closely related to bone and cartilage loss in $O A$, and many studies have demonstrated that the MMP induction by IL- $1 \beta$ is mediated by the ERK-MAPK signalling pathway $[24,25$, 26]. ERK $1 / 2$ is involved in a variety of cellular responses in bone and cartilage and is one of the important members of the MAPK cascade. It is well known that VEGF, a classical angiogenic factor, is closely related to the progression of OA disease. It has been confirmed that when VEGF is combined with its signal receptor VEGF2, downstream kinases such as MAPK can be phosphorylated to activate the ERKMAPK signalling pathway [27]. Moreover, ERK-MAPK signalling pathway activated by VEGF can induce the activation of MMPs [28]. Because VEGF, MMPs, and the ERK-MAPK pathways are closely related to the development of $O A$ and there is a close relationship between them, they are regarded as the $O A$ phenotypes in our vitro experiments.

MicroRNAs determine cell fate by participating in the regulation of extracellular signalling pathways and expression of certain molecules [29], and miR-21-5p has been reported to play an important role in cell apoptosis, pathological growth and stress [30,31], processes that are closely related to OA cartilage lesions. Some researchers have found that miR-21-5p plays an important role in the development of inflammation [29, 30,31], and there have been many studies on the involvement of miR-21-5p in the progression of various diseases through its target genes Spry 1 and Spry2 [15, 29, 35]. More importantly, 
Wang et al. [13] and Zhang et al. [14] have recently demonstrated that miR-21-5p promotes the progression of OA by targeting growth differentiation factor 5 (GDF5) and FGF18, respectively. At present, there have been no studies on the regulatory mechanism of miR-21-5p and its target gene Spry 1 in OA. However, many research groups have confirmed that Spry1 can inhibit the phosphorylation of the ERKMAPK signalling pathway and the expression of $\operatorname{VEGF}[15,16,36,37,38,39]$. Since miR-21-5p, ERKMAPK and VEGF are closely related to OA, we hypothesized that Spry 1 is likely to act as a link between miR-21-5p, ERK-MAPK and VEGF and to play a regulatory role in the development of TMJOA. We established a UAC model that can induce TMJOA in vivo. The staining results suggest that KO miR-21-5p can delay or even inhibit the development of TMJOA disease induced by surgery. The dual luciferase assay results showed that miR-21-5p could also directly target the expression of Spry 1 in cartilage. Then, by transfecting MCCs with miR-21-5p mimic we demonstrated that upregulation of miR-21-5p inhibited the expression of Spry1 protein and promoted the secretion of VEGF and MMP-13 and the phosphorylation of ERK-MAPK pathway components. Conversely, inhibition of miR-21-5p had the opposite effects. We induced the inflammatory state of MCCs by IL-1 $\beta$ and found that miR-21-5p inhibitor could attenuate the effect of IL-1 $\beta$ on the expression of VEGF, MMP-13, p-ERK1/2 and upregulate the expression of the target gene Spry 1 of miR-21-5p, while overexpression of miR-21-5p had the opposite effect. It is suggested that miR-21-5p may be involved in the catabolism of MCCs by targeting Spry1. The positive correlation between miR-21-5p and OA is also consistent with the conclusions of Wang et al. [13] and Zhang et al. [14]. To further clarify that miR-21-5p promotes the secretion of VEGF and MMP-13 and phosphorylation of ERK-MAPK signalling pathway components by targeting Spry1, we co-transfected MCCs with si-Spry1 and miR-21-5p inhibitor or with cDNA-Spry1 and miR-21-5p inhibitor. Interestingly, overexpression of Spry1 enhanced the protective effect of miR-21-5p inhibitor on TMJOA. These results indicate that miR-21-5p promotes the degradation of the extracellular matrix by targeting Spry 1 to upregulate the expression of VEGF and MMP-13 and phosphorylation of ERK-MAPK signalling pathway components in inflammatory MCCs. It is worth noting that there is a significant positive correlation between VEGF and miR-21-5p in vitro. Moreover, according to many studies, miR-21-5p can regulate the phosphorylation of ERK-MAPK signalling pathway components by targeting Spry 1 in a variety of cells $[15,35,39,40]$, and based on the close correlation between VEGF and the ERK-MAPK signalling pathway $[7,28,39]$, we speculate that miR-21-5p can promote angiogenesis in TMJ, and this effect is likely related to the degree of phosphorylation of ERK-MAPK signalling pathway components. Finally, the experimental results of the chicken embryo angiogenesis assay and Western blotting showed that miR-21-5p has a proangiogenic effect, which was significantly mediated by the ERK-MAPK signalling pathway.

This experiment only verified that miR-21-5p targeting Spry 1 can simultaneously increase the expression of VEGF, MMP-13 and p-ERK1/2. However, in this signalling axis, whether VEGF is upstream or downstream of ERK-MAPK and whether VEGF has a feedback promoting or inhibiting effect on the expression of Spry 1 need further experimental verification. In addition, due to experimental constraints, we could not obtain enough human TMJ samples in a short time, which is a clinical limitation of our study. 


\section{Conclusions}

To the best of our knowledge, this is the first report of miR-21-5p affecting the progression of TMJOA disease in mice by targeting Spry1. We concluded that KO miR-21-5p attenuates the deterioration of condylar cartilage structure and function caused by the mouse UAC model. Furthermore, in the inflammatory state induced by IL-1 $\beta$, miR-21-5p can promote the degradation of extracellular matrix and angiogenesis in TMJOA by downregulating the expression of Spry1. These findings not only provide new insights into the role of Spry1, a classical inhibitor of the Ras/Raf/ERK signalling pathway, in regulating TMJOA matrix degradation and angiogenesis but also provide a new strategy for the future use of miRNAs in TMJOA treatment.

\section{Abbreviations}

TMJOA: Temporomandibular joint osteoarthritis; TMJ: Temporomandibular joint; MiRs: MicroRNAs; Spry1: Sprouty1; UAC: Unilateral anterior crossbite; CAM: Chick embryo chorioallantoic membrane; MCCs: Mandibular condylar chondrocytes; ACAN: Aggrecan; VEGF: Vascular endothelial growth factor; MMPs: Matrix metalloproteinases; 3'UTR: 3'- untranslated region; FGF18: Fibroblast growth factor 18; KO: Knockout; MiR-21-5p KO : MiR-21-5p ${ }^{-/-}$C57BL/6 knock-out; MiR-21-5p: MicroRNA-21-5p; WT: Wild-type; NC: Negative control; IOD: Integrated Optical Density; AREA: The area of the pixel; BSA: Bovine serum albumin; mimic NC : Mimic negative control ; inhibitor NC : Inhibitor negative control ; si-Spry1: Small interference RNA of mouse Spry1; si-NC: Negative control siRNA; cDNA Spry1: ectopic expression plasmid of Spry1; CT: Comparative threshold cycle; GADPH: Glyceraldehyde- 3-phosphate dehydrogenase; DAPI: 4', 6-diamidino-2-phenylindole; SEML: Standard error of the mean; ANOVA: one-way analysis of variation; IHC: immunohistochemistry; qRT-PCR: Real-time quantitative reverse transcriptase polymerase chain reaction; GDF5: growth differentiation factor 5.

\section{Declarations}

\section{Acknowledgement}

We thank the anonymous reviewer's suggestions and comments.

\section{Funding}

This work was supported by National Natural Science Foundation of China (61771290, 61871393), Taishan Scholars (tsqn201812137), China Postdoctoral Science Foundation (2019M652408), Natural Science Foundation of Shandong Province (ZR2018PH022, ZR2019PH015), The Fundamental Research Funds of Shandong University (2019GN091) and The Science and Technology Development Plans of Shandong province (2018GSF118196).

\section{Availability of data and materials}


The datasets used and/or analyzed during the study are available from the corresponding author on reasonable request.

\section{Author contributions}

Designed the research, and drafted the manuscript: Shixing Ma, Aobo Zhang, Shaopeng Liu, Xiaole Li

Conducted the experiments: Shixing Ma and Aobo Zhang

Collected and analyzed the data: Shixing Ma and Lie Chen

Contributed analysis tools materials: Huaqiang Zhao, Lei Chen, Chuan Ma, Shizhou Zhang.

\section{Ethics approval and consent to participate}

According to the recommendations of Chinese Academy of Sciences, the protocol of this study was authorized by the Animal Experimental Ethics Committee of Shandong University. Informed consent was signed by all participants.

\section{Consent for publication}

Not applicable.

\section{Competing interests}

The authors declare that they have no competing interests.

\section{Author details}

$1,7,8$ Shandong Provincial Key Laboratory of Oral Tissue Regeneration \& Department of Oral and Maxillofacial Surgery, School of Stomatology, Shandong University, Jinan, Shandong, China. 2,6 Shandong Provincial Key Laboratory of Oral Tissue Regeneration \& Department of Orthodontics, School of Stomatology, Shandong University, Jinan, Shandong, China. ${ }^{3}$ Department of Oral and Maxillofacial Surgery, Shandong Provincial Hospital Affiliated to Shandong University, Jinan, Shandong 250021, P.R. China. 4Ningbo Stomatology Hospital. Ningbo 315010, Zhejiang Province, China. ${ }^{5}$ The Institute for Tissue Engineering and Regenerative, Liaocheng People's Hospital, Liaocheng, Shandong, China.

\section{References}

1. Tanaka E, Detamore MS, Mercuri LG: Degenerative disorders of the temporomandibular joint: etiology, diagnosis, and treatment. J Dent Res 2008, 87(4):296-307. 
2. Li K, Zhang Y, Zhang Y, Jiang W, Shen J, Xu S, Cai D, Shen J, Huang B, Li M et al: Tyrosine kinase Fyn promotes osteoarthritis by activating the beta-catenin pathway. Annals of the rheumatic diseases 2018, 77(6):935-943.

3. Miyaki S, Asahara H: Macro view of microRNA function in osteoarthritis. Nature reviews Rheumatology 2012, 8(9):543-552.

4. Walsh DA, Haywood L: Angiogenesis: a therapeutic target in arthritis. Current opinion in investigational drugs (London, England : 2000) 2001, 2(8):1054-1063..

5. Shen P, Jiao Z, Zheng JS, Xu WF, Zhang SY, Qin A, Yang C: Injecting vascular endothelial growth factor into the temporomandibular joint induces osteoarthritis in mice. Sci Rep 2015, 5:16244.

6. Leonardi R, Lo Muzio L, Bernasconi G, Caltabiano C, Piacentini C, Caltabiano M: Expression of vascular endothelial growth factor in human dysfunctional temporomandibular joint discs. Arch Oral Biol 2003, 48(3):185-192.

7. Pufe T, Harde V, Petersen W, Goldring MB, Tillmann B, Mentlein R: Vascular endothelial growth factor (VEGF) induces matrix metalloproteinase expression in immortalized chondrocytes. The Journal of pathology 2004, 202(3):367-374.

8. Prasadam I, Crawford R, Xiao Y: Aggravation of ADAMTS and matrix metalloproteinase production and role of ERK1/2 pathway in the interaction of osteoarthritic subchondral bone osteoblasts and articular cartilage chondrocytes -- possible pathogenic role in osteoarthritis. The Journal of rheumatology 2012, 39(3):621-634.

9. Farh KK, Grimson A, Jan C, Lewis BP, Johnston WK, Lim LP, Burge CB, Bartel DP: The widespread impact of mammalian MicroRNAs on mRNA repression and evolution. Science (New York, NY) 2005, 310(5755):1817-1821.

10. West C, McDermott MF: Effects of microRNA-146a on the proliferation and apoptosis of human osteochondrocytes by targeting TRAF6 through the NF- kappaB signalling pathway. Bioscience reports 2017, 37(4).

11. Seidl Cl, Martinez-Sanchez A, Murphy CL: Derepression of MicroRNA-138 Contributes to Loss of the Human Articular Chondrocyte Phenotype. Arthritis \& rheumatology (Hoboken, NJ) 2016, 68(2):398409.

12. Tu M, Li Y, Zeng C, Deng Z, Gao S, Xiao W, Luo W, Jiang W, Li L, Lei G: MicroRNA-127-5p regulates osteopontin expression and osteopontin-mediated proliferation of human chondrocytes. Sci Rep $2016,6: 25032$.

13. Wang XB, Zhao FC, Yi LH, Tang JL, Zhu ZY, Pang Y, Chen YS, Li DY, Guo KJ, Zheng X: MicroRNA-21$5 p$ as a novel therapeutic target for osteoarthritis. Rheumatology (Oxford, England) 2019.

14. Zhang Y, Jia J, Yang S, Liu X, Ye S, Tian H: MicroRNA-21 controls the development of osteoarthritis by targeting GDF-5 in chondrocytes. Experimental \& molecular medicine 2014, 46:e79.

15. Thum T, Gross C, Fiedler J, Fischer T, Kissler S, Bussen M, Galuppo P, Just S, Rottbauer W, Frantz S et al: MicroRNA-21 contributes to myocardial disease by stimulating MAP kinase signalling in fibroblasts. Nature 2008, 456(7224):980-984.

Page $14 / 26$ 
16. Guy GR, Jackson RA, Yusoff P, Chow SY: Sprouty proteins: modified modulators, matchmakers or missing links? The Journal of endocrinology 2009, 203(2):191-202.

17. Impagnatiello MA, Weitzer S, Gannon G, Compagni A, Cotten M, Christofori G: Mammalian sprouty-1 and -2 are membrane-anchored phosphoprotein inhibitors of growth factor signaling in endothelial cells. The Journal of cell biology 2001, 152(5):1087-1098.

18. Alakoski T, Ulvila J, Yrjola R, Vainio L, Magga J, Szabo Z, Licht JD, Kerkela R: Inhibition of cardiomyocyte Sprouty 1 protects from cardiac ischemia-reperfusion injury. Basic research in cardiology $2019,114(2): 7$.

19. Lu L, Huang J, Zhang X, Zhang J, Zhang M, Jing L, Yu S, Wang M: Changes of temporomandibular joint and semaphorin 4D/Plexin-B1 expression in a mouse model of incisor malocclusion. $J$ Oral Facial Pain Headache 2014, 28(1):68-79.

20. Zhang HY, Liu YD, Yang HX, Zhang M, Liao LF, Wan XH, Wang MQ: Installing and thereafter removing an aberrant prosthesis elicited opposite remodelling responses in growing mouse temporomandibular joints. J Oral Rehabil 2015, 42(9):685-692.

21. Lu L, Zhang X, Zhang M, Zhang H, Liao L, Yang T, Zhang J, Xian L, Chen D, Wang M: RANTES and SDF-1 Are Keys in Cell-based Therapy of TMJ Osteoarthritis. J Dent Res 2015, 94(11):1601-1609.

22. Liu Q, Yang HX, Wan XH, Zhang M, Zhang J, Lu L, Xie M, Ren HT, Yu SB, Liu XD et al: Calcium-/calmodulin-dependent protein kinase II in occlusion-induced degenerative cartilage of rat mandibular condyle. J Oral Rehabil 2018, 45(6):442-451.

23. Sylvester J, El Mabrouk M, Ahmad R, Chaudry A, Zafarullah M: Interleukin-1 induction of aggrecanase gene expression in human articular chondrocytes is mediated by mitogen-activated protein kinases. Cellular physiology and biochemistry : international journal of experimental cellular physiology, biochemistry, and pharmacology 2012, 30(3):563-574.

24. Liacini A, Sylvester J, Li WQ, Zafarullah M: Inhibition of interleukin-1-stimulated MAP kinases, activating protein-1 (AP-1) and nuclear factor kappa B (NF-kappa B) transcription factors downregulates matrix metalloproteinase gene expression in articular chondrocytes. Matrix biology : journal of the International Society for Matrix Biology 2002, 21(3):251-262.

25. Shum JK, Melendez JA, Jeffrey JJ: Serotonin-induced MMP-13 production is mediated via phospholipase C, protein kinase C, and ERK1/2 in rat uterine smooth muscle cells. The Journal of biological chemistry 2002, 277(45):42830-42840.

26. Wei Y, Zhi-Hong W, Gui-Xing Q, Bin Y, Jun C, Yi-Peng W: Extracellular signal-regulated kinase inhibition modulates rat annulus fibrosus cell response to interleukin-1. Spine 2013, 38(17):E1075-1081.

27. Neufeld G, Cohen T, Gengrinovitch S, Poltorak Z: Vascular endothelial growth factor (VEGF) and its receptors. FASEB journal : official publication of the Federation of American Societies for Experimental Biology 1999, 13(1):9-22.

28. Dong Y, Wu G, Zhu T, Chen H, Zhu Y, Zhu G, Han F, Zhao H: VEGF promotes cartilage angiogenesis by phospho-ERK1/2 activation of DII4 signaling in temporomandibular joint osteoarthritis caused by chronic sleep disturbance in Wistar rats. Oncotarget 2017, 8(11):17849-17861. 
29. Mei Y, Bian C, Li J, Du Z, Zhou H, Yang Z, Zhao RC: miR-21 modulates the ERK-MAPK signaling pathway by regulating SPRY2 expression during human mesenchymal stem cell differentiation. Journal of cellular biochemistry 2013, 114(6):1374-1384.

30. Chan JA, Krichevsky AM, Kosik KS: MicroRNA-21 is an antiapoptotic factor in human glioblastoma cells. Cancer Res 2005, 65(14):6029-6033.

31. Bondi ML, Ferraro M, Di Vincenzo S, Gerbino S, Cavallaro G, Giammona G, Botto C, Gjomarkaj M, Pace E: Effects in cigarette smoke stimulated bronchial epithelial cells of a corticosteroid entrapped into nanostructured lipid carriers. Journal of nanobiotechnology 2014, 12:46.

32. Olivieri F, Spazzafumo L, Santini G, Lazzarini R, Albertini MC, Rippo MR, Galeazzi R, Abbatecola AM, Marcheselli F, Monti D et al: Age-related differences in the expression of circulating microRNAs: miR21 as a new circulating marker of inflammaging. Mechanisms of ageing and development 2012, 133(11-12):675-685.

33. Ciancio G, Ferracin M, Saccenti E, Bagnari V, Farina I, Furini F, Galuppi E, Zagatti B, Trotta F, Negrini M et al: Characterisation of peripheral blood mononuclear cell microRNA in early onset psoriatic arthritis. Clinical and experimental rheumatology 2017, 35(1):113-121.

34. Dong L, Wang X, Tan J, Li H, Qian W, Chen J, Chen Q, Wang J, Xu W, Tao C et al: Decreased expression of microRNA-21 correlates with the imbalance of Th17 and Treg cells in patients with rheumatoid arthritis. Journal of cellular and molecular medicine 2014, 18(11):2213-2224.

35. Ning ZW, Luo XY, Wang GZ, Li Y, Pan MX, Yang RQ, Ling XG, Huang S, Ma XX, Jin SY et al: MicroRNA21 Mediates Angiotensin II-Induced Liver Fibrosis by Activating NLRP3 Inflammasome/IL-1beta Axis via Targeting Smad7 and Spry1. Antioxidants \& redox signaling 2017, 27(1):1-20.

36. Huebert RC, Li Q, Adhikari N, Charles NJ, Han X, Ezzat MK, Grindle S, Park S, Ormaza S, Fermin D et al: Identification and regulation of Sprouty 1 , a negative inhibitor of the ERK cascade, in the human heart. Physiological genomics 2004, 18(3):284-289.

37. Li X, Wheldon L, Heath JK: Sprouty: a controversial role in receptor tyrosine kinase signalling pathways. Biochemical Society transactions 2003, 31(Pt 6):1445-1446.

38. Edwin F, Anderson K, Ying C, Patel TB: Intermolecular interactions of Sprouty proteins and their implications in development and disease. Molecular pharmacology 2009, 76(4):679-691.

39. Haque R, luvone PM, He L, Choi KSC, Ngo A, Gokhale S, Aseem M, Park D: The MicroRNA-21 signaling pathway is involved in prorenin receptor (PRR) -induced VEGF expression in ARPE-19 cells under a hyperglycemic condition. Molecular vision 2017, 23:251-262.

40. Ma X, Kumar M, Choudhury SN, Becker Buscaglia LE, Barker JR, Kanakamedala K, Liu MF, Li Y: Loss of the miR-21 allele elevates the expression of its target genes and reduces tumorigenesis. Proceedings of the National Academy of Sciences of the United States of America 2011, 108(25):10144-10149. 


\section{Tables}

TABLE 1 Transfection sequences for miR-21-5p mimic, miR-21-5p inhibitor, siRNA-Spry1

\begin{tabular}{|l|l|}
\hline Gene & Sequence(5'-3') \\
\hline miR-21-5p mimic & UAGCUUAUCAGAC UGAUGUUGA \\
\hline miR-21-5p inhibitor & UAGCUUAUCAGACUGAUGUUGA \\
\hline siRNA-Spry1 & CCCAGAATGTTGACAGCTGCCTCTT \\
\hline
\end{tabular}

Note. miR-21-5p: microRNA-21-5p; siRNA: small interfering RNA

Table 2 Number of vascular branches in different groups

\begin{tabular}{ccccc}
\hline Embryo number $^{\#}$ & NC & Inhibitor & mimic & mimic+U0126 \\
\hline $1^{\#}$ & 159 & 217 & 289 & 138 \\
$2^{\#}$ & 220 & 182 & 270 & 187 \\
\hline $3^{\#}$ & 244 & 197 & 291 & 178 \\
\hline $4^{\#}$ & 249 & 212 & 290 & 210 \\
\hline $5^{\#}$ & 269 & 207 & 260 & 168 \\
$6^{\#}$ & 225 & 112 & 316 & 137 \\
\hline $7^{\#}$ & 286 & 127 & 287 & 148 \\
\hline $8^{\#}$ & 239 & 162 & 299 & 198 \\
\hline Mean \pm standard deviation & $236.38 \pm 38.05$ & $188.25 \pm 36.23$ & $287.75 \pm 15.9$ & $170.50 \pm 27.61$ \\
\hline
\end{tabular}

Note. inhibitor: microRNA-21-5p inhibitor; mimic: microRNA-21-5p mimic

\section{Figures}



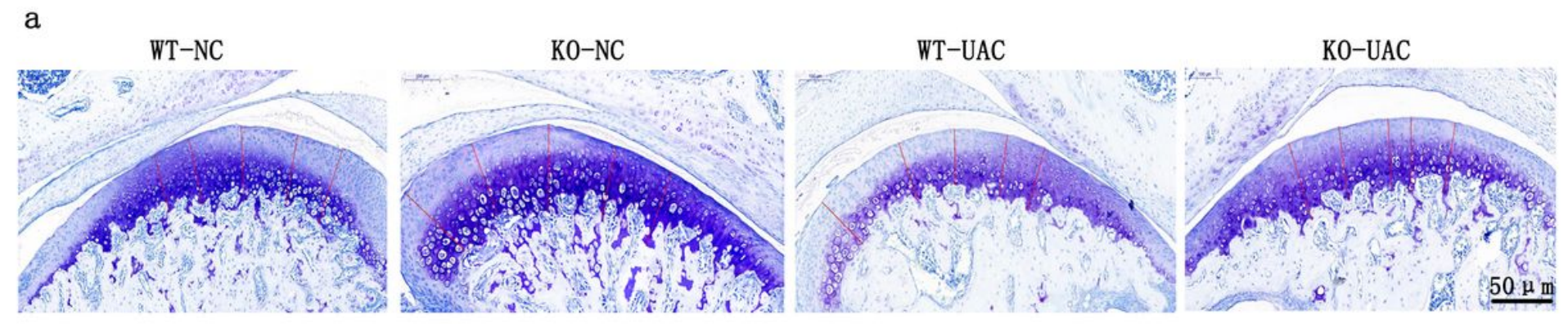

$\mathrm{b}$

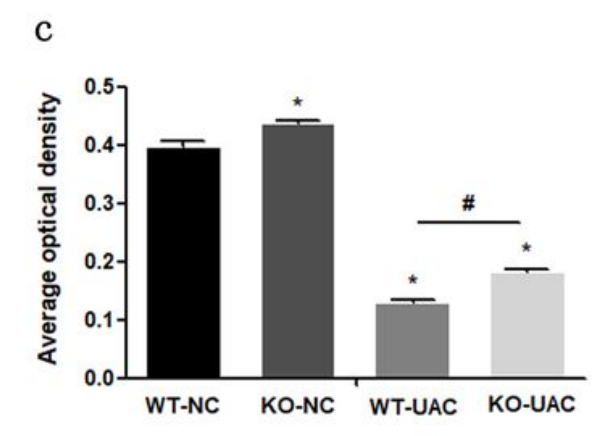

$\mathrm{d}$

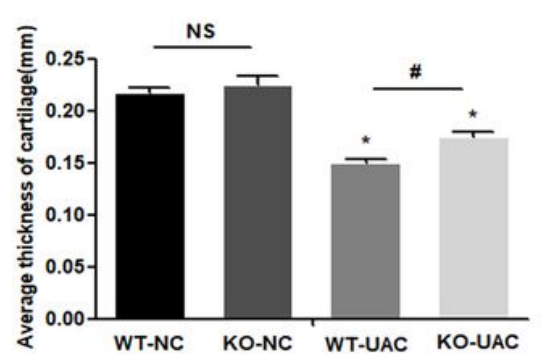

WT-NC

$\mathrm{K} 0-\mathrm{NC}$

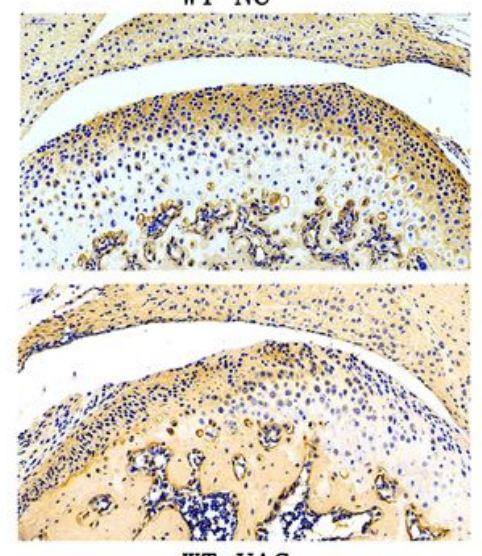

WT-UAC

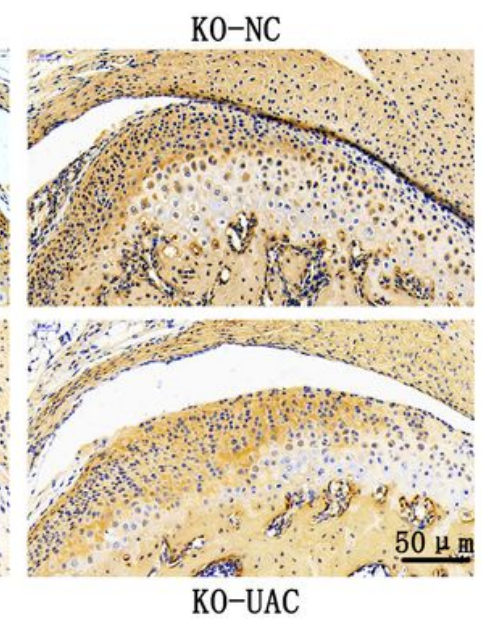

e

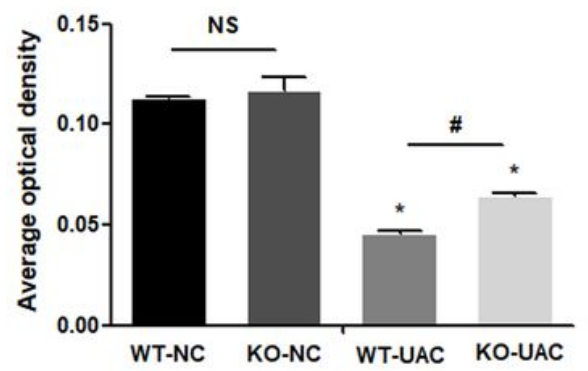

Figure 1

Toluidine blue (TB) and immunohistochemical (IHC) staining images of each section are shown. a, b, c, d Results showing that severe cartilage degeneration and loss of aggrecan (ACAN) of cartilage occurred in the unilateral anterior crossbite (UAC) group compared with the NC control group. In the UAC groups, the knockout (KO) UAC group had a reversal effect on cartilage degradation and ACAN loss. Comparison of the average optical density (AOD) (b) and the thickness of cartilage (c) of TB staining between groups. e Comparison of the AOD of IHC staining for ACAN between groups. Asterisk (*): compared with the wildtype negative control (WT-NC) group. Hash (\#): compared with the wild-type UAC (WT-UAC) group. Data are represented as the means \pm standard deviation $(n=3) .{ }^{*} P<0.05, \# P<0.05$. NS: not significant. Scale bar: $50 \mu \mathrm{m}$. 

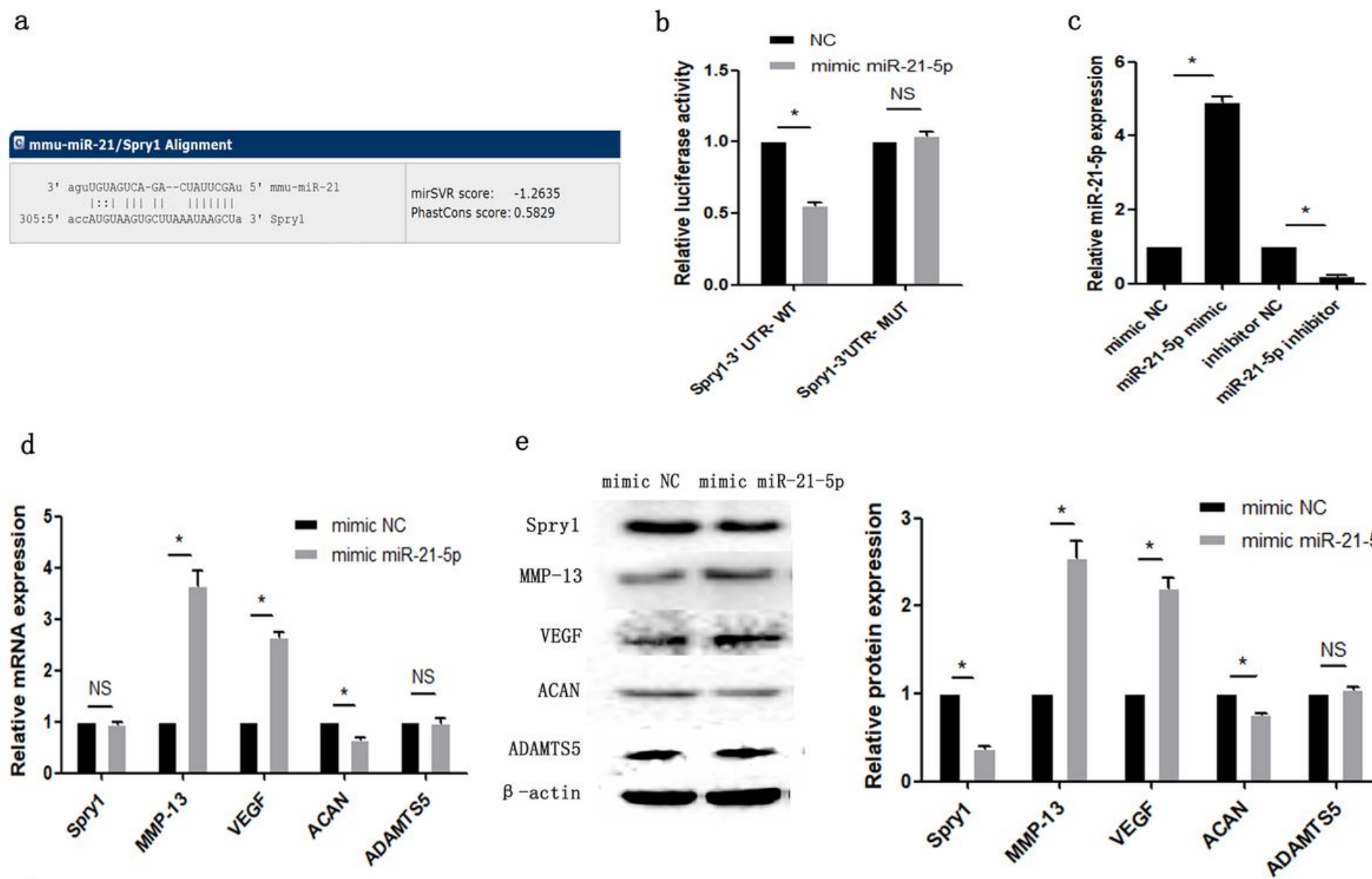

$\mathrm{e}$
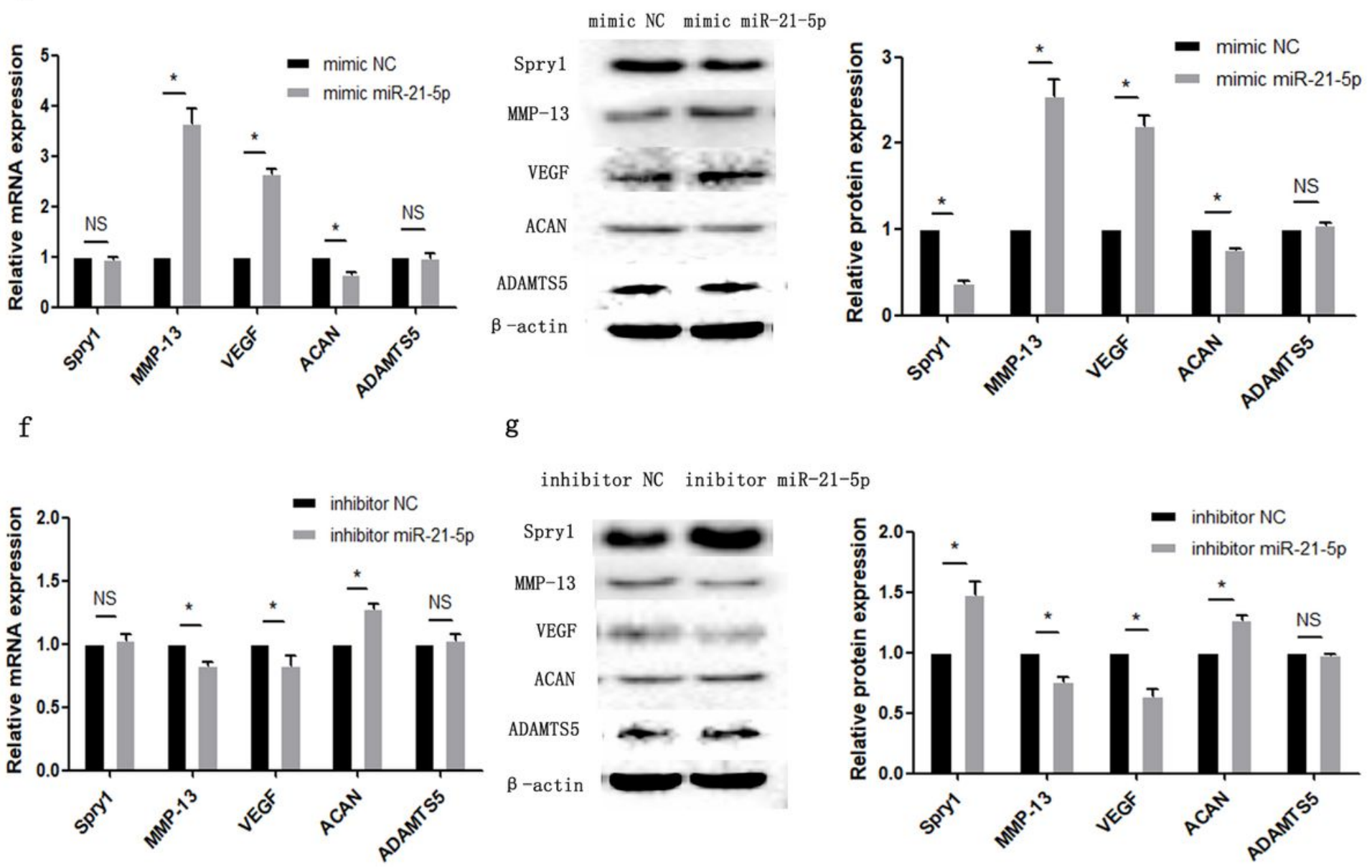

g

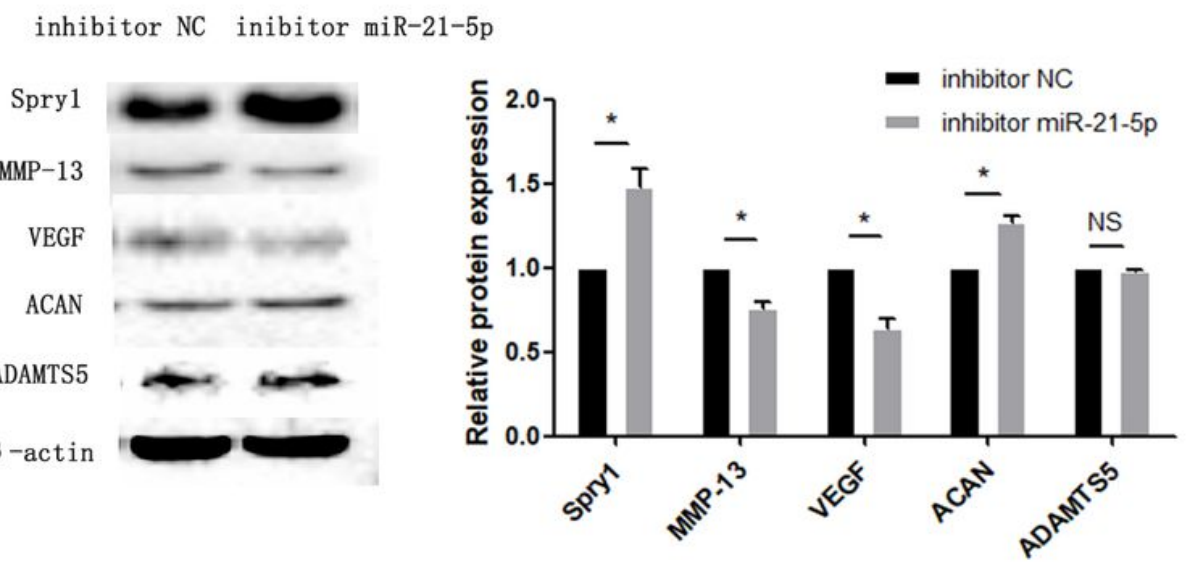

Figure 2

MiR-21-5p directly regulates the expression of Spry1 and affects the expression of specific genes in mandibular condylar chondrocytes (MCCs). a The predicted binding sites of miR-21-5p to the 3' UTR of Spry1. b Dual luciferase reporter assay showing that Spry1 is the target gene of miR-21-5p. Asterisk (*): compared with the negative control (NC) group. c Evaluation of the relative microRNA expression of miR21-5p in MCCs transfected with mimic or inhibitor. RT-qPCR (d) and Western blotting (e) results of cartilage-specific gene expression in MCCs transfected with miR-21-5p mimic. RT-qPCR (f) and Western blotting (g) results of cartilage-specific gene expression in MCCs transfected with miR-21-5p inhibitor. 
Asterisk (*): compared with the negative control (NC) group. Data are represented as the means \pm standard deviation $(n=3)$. ${ }^{*} P<0.05$. NS: not significant.

a

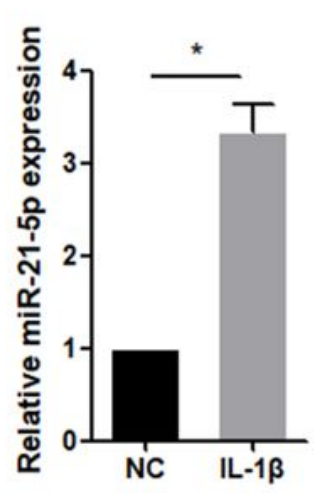

C

$\begin{array}{rcccc}\mathrm{IL}-1 \mathrm{\beta}(10 \mathrm{ng} / \mathrm{ml}) & - & - & + & + \\ \operatorname{mimic~NC} & + & - & + & - \\ \operatorname{mimic} \mathrm{miR}-21-5 \mathrm{p} & - & + & - & +\end{array}$

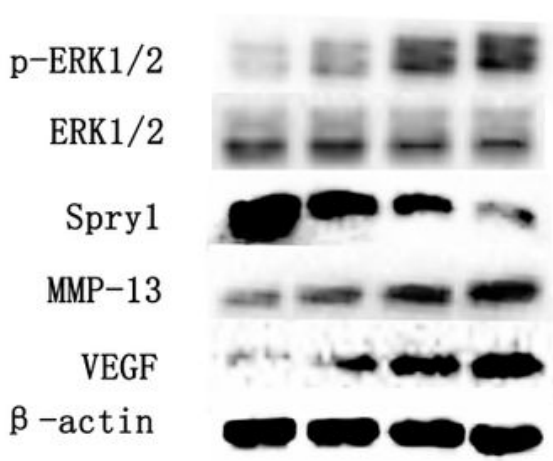

$\mathrm{d}$

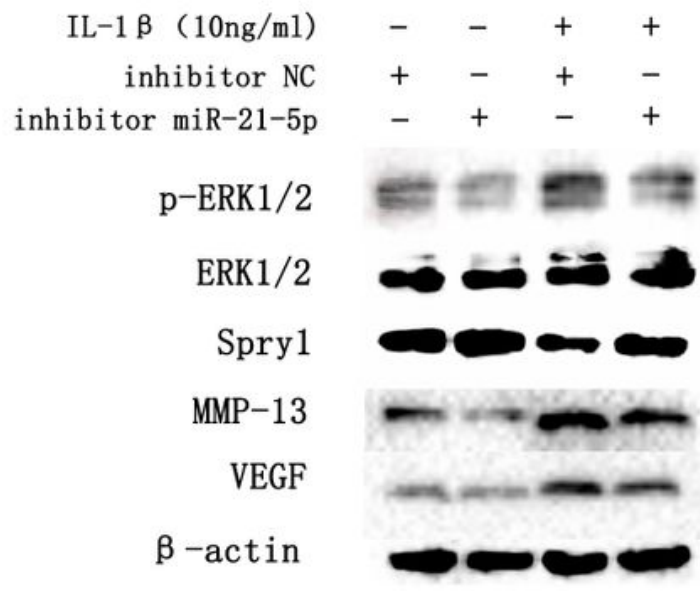

b
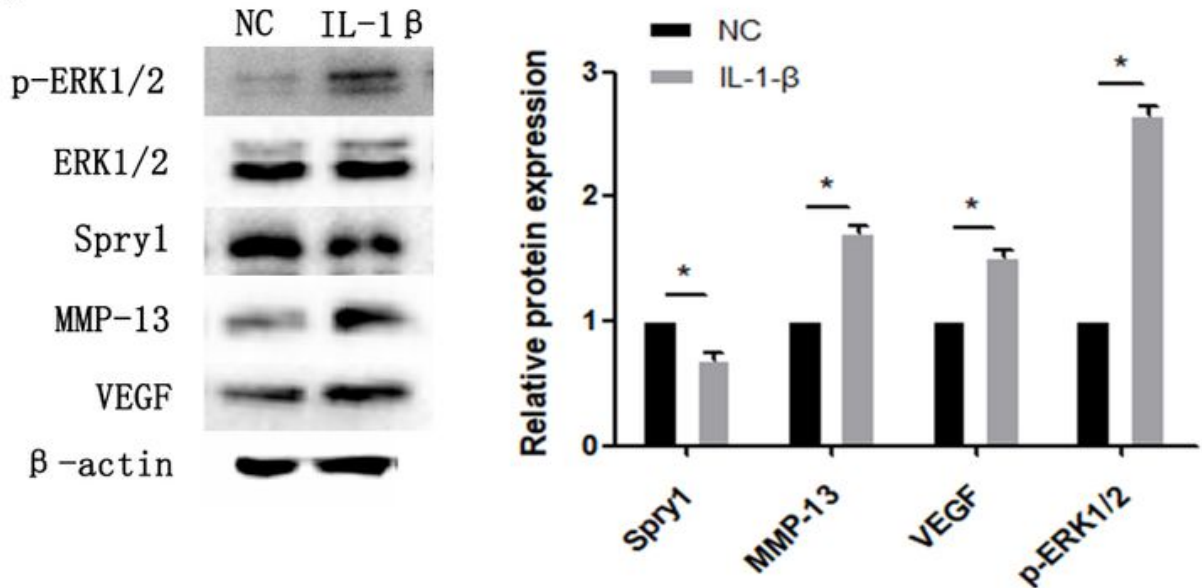

- mimic NC

mimic miR-21-5p

- mimic NC+IL-1 $\beta$

$=\quad$ mimic miR-21-5p+IL-1 $\beta$
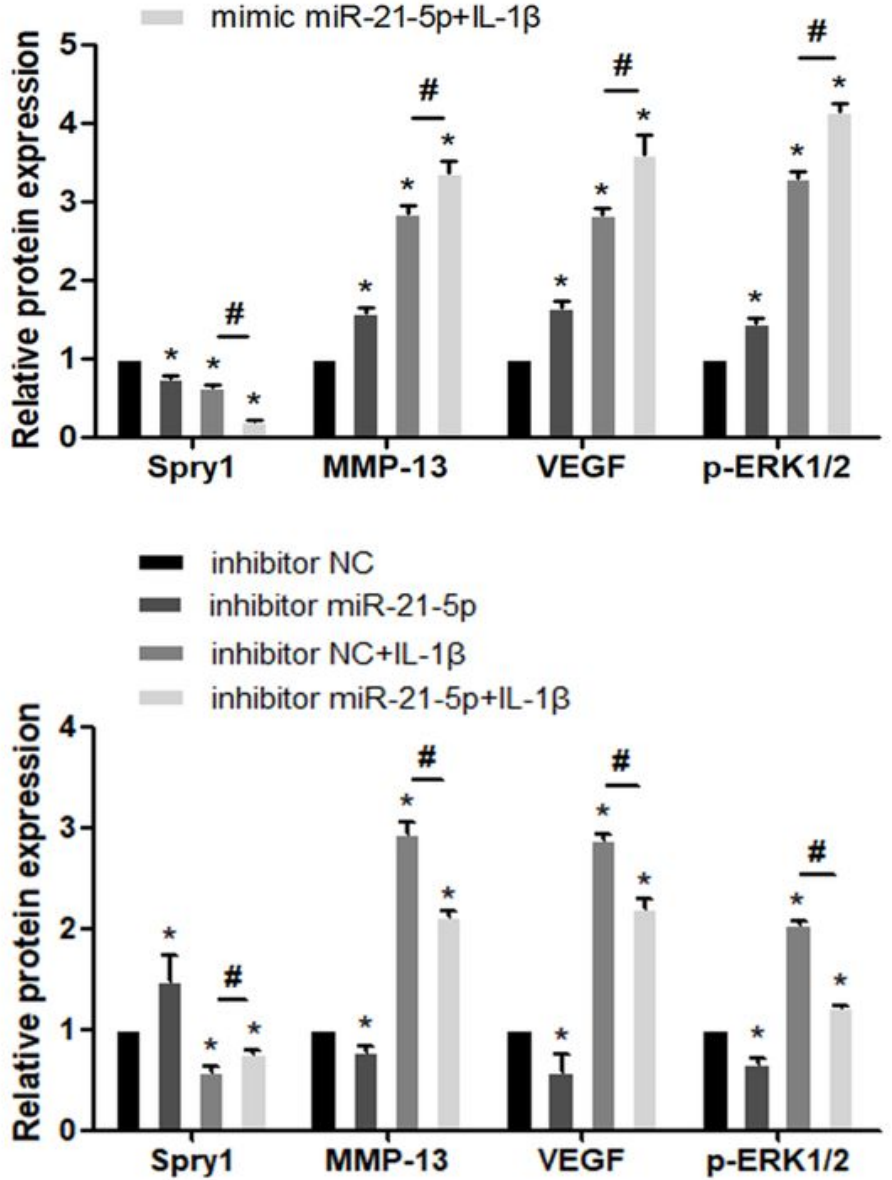

\section{Figure 3}

MiR-21-5p mediates the expression of related genes in IL-1 $\beta$-induced condylar chondrocytes. a RT-qPCR detected the expression of miR-21-5p. b Protein expression levels of Spry1, MMP-13, VEGF, and p-ERK1/2 were assayed by Western blot in MCCs under stimulation with IL-1 $\beta$ for $24 \mathrm{~h}$. Western blotting results of 
Spry1, MMP-13, VEGF, and p-ERK1/2 proteins in MCCs transfected with miR-21-5p mimic (c) or miR-21-5p inhibitor (d), followed by stimulation with IL-1 $\beta$ for $24 \mathrm{~h}$. Asterisk( $\left.{ }^{*}\right)$ : compared with mimic NC (b, d) or inhibitor NC group (c, e). Hash (\#): compared with the mimic NC+ IL-1 $\beta$ or inhibitor NC+IL-1 $\beta$ group. Data are represented as the means \pm standard deviation $(n=3)$. ${ }^{*}<0.05$, \#P<0.05. NS: not significant.

a
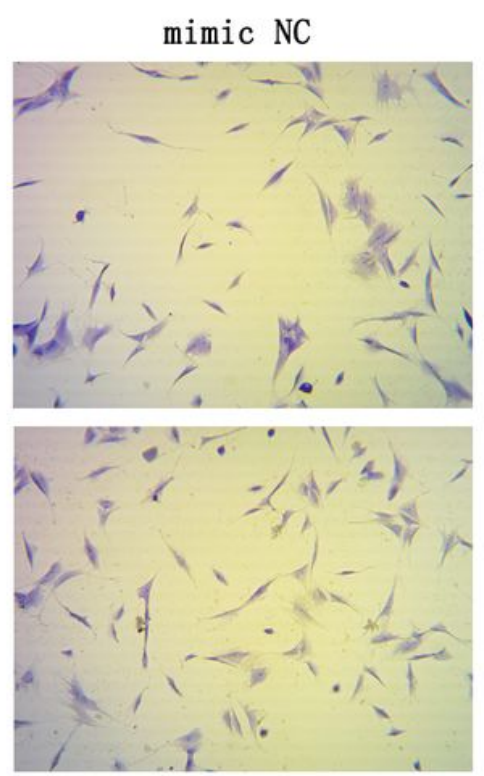

IL-1 $\beta+$ mimic NC

$\mathrm{C}$
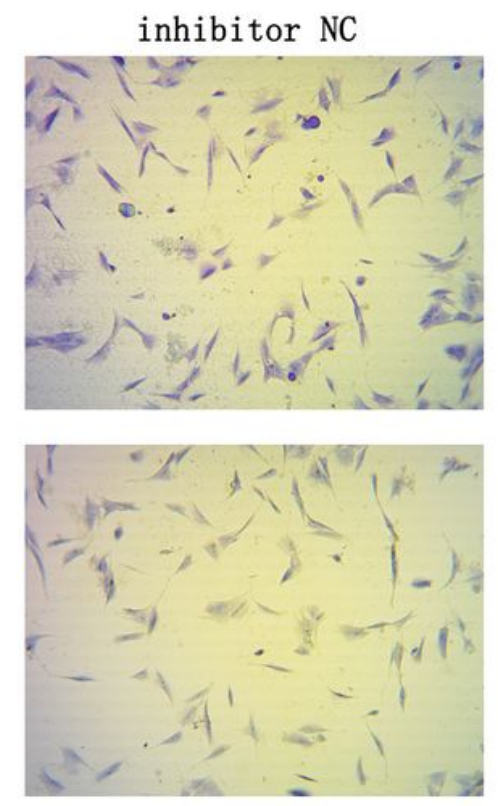

IL-1 $\beta+$ inhibitor NC
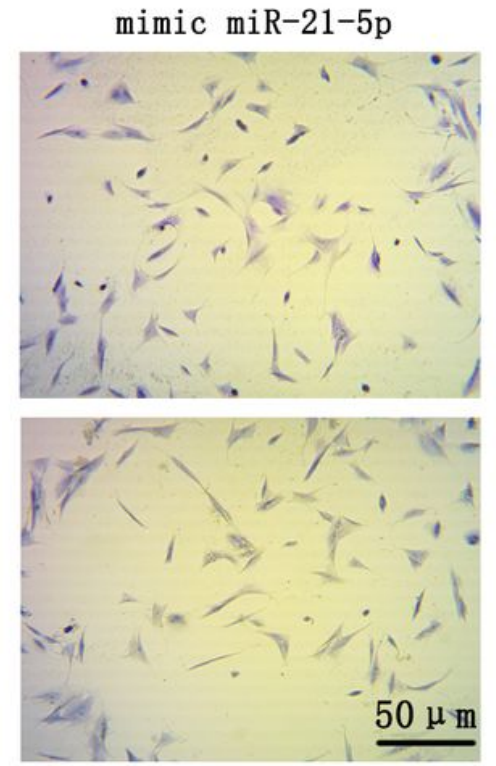

IL-1 $\beta+$ mimic miR-21-5p inhibitor miR-21-5p
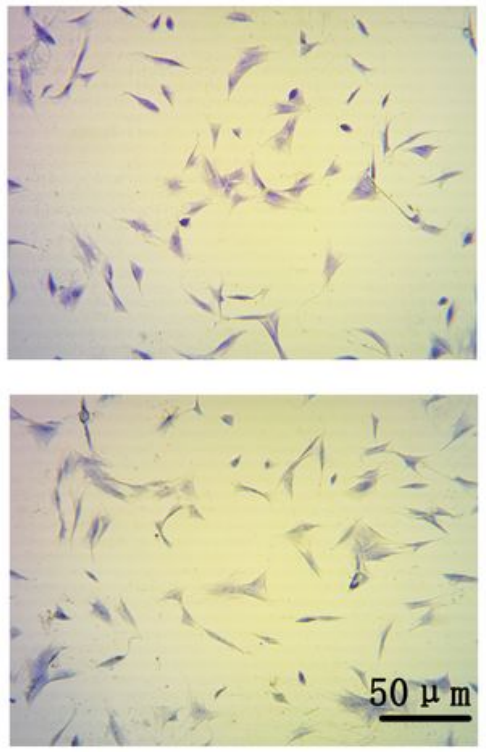

IL-1 $\beta$ +inhibitor miR-21-5p $\mathrm{b}$

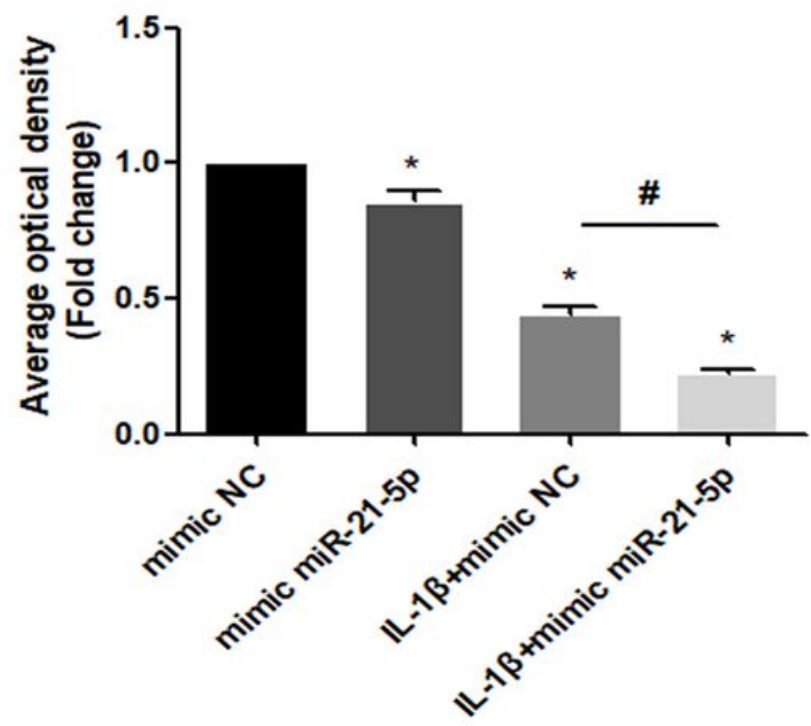

d

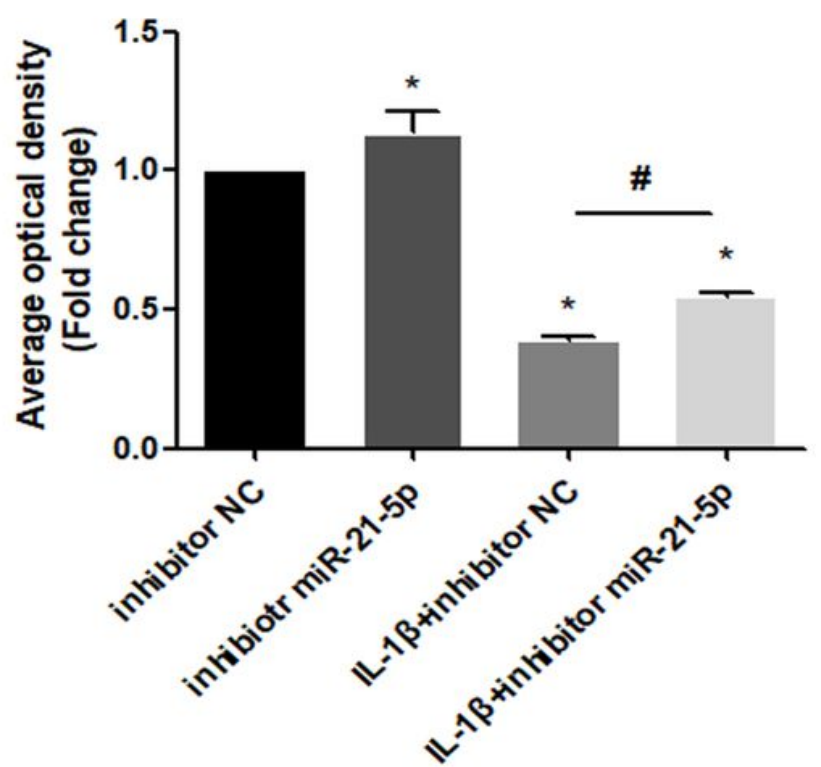

\section{Figure 4}

Toluidine blue staining results of MCCs transfected with miR-21-5p mimic (a) or miR-21-5p inhibitor (c), followed by stimulation with IL-1 $\beta$ for $24 \mathrm{~h}$. Scale bar, $50 \mu \mathrm{m}$ (magnification, $\times 200$ ). Lower panel, 
statistical analysis of the average optical density of matrix staining by toluidine blue. Asterisk (*): compared with the mimic NC (b) or inhibitor NC group (d). Hash (\#): compared with the mimic NC+ IL-1 $\beta$ or inhibitor NC+ IL-1 $\beta$ group. Data are represented as the means \pm standard deviation $(n=3)$. ${ }^{*}<0.05$, \#P $<0.05$. NS: not significant. Scale bar: $50 \mu \mathrm{m}$.

a

NC

mimic

$\operatorname{miR}-21-5 p$

inhibitor

miR-21-5p

$\mathrm{IL}-1 \beta+\mathrm{NC}$

$\mathrm{IL}-1 \beta+$ mimic

miR-21-5p

IL-1 $\beta$ +inhibitor miR-21-5p
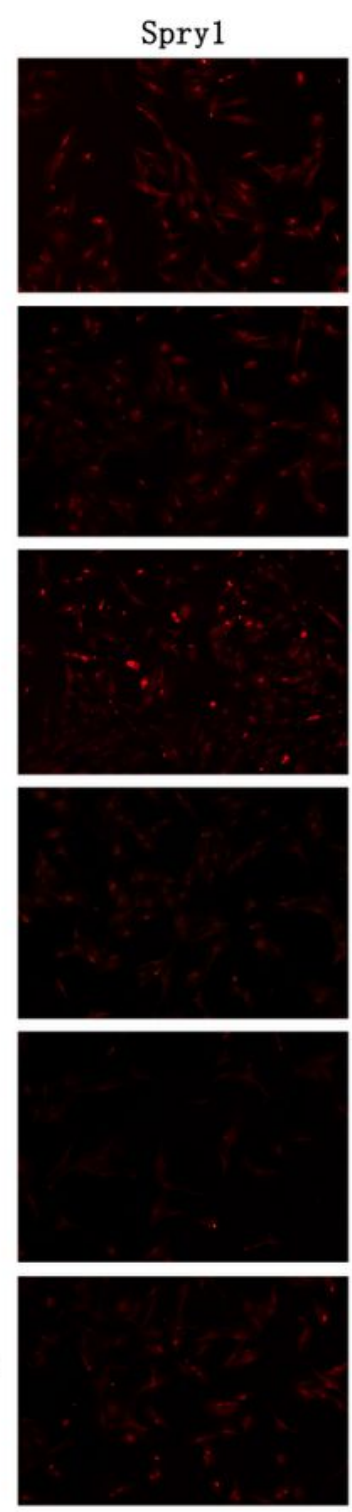

b

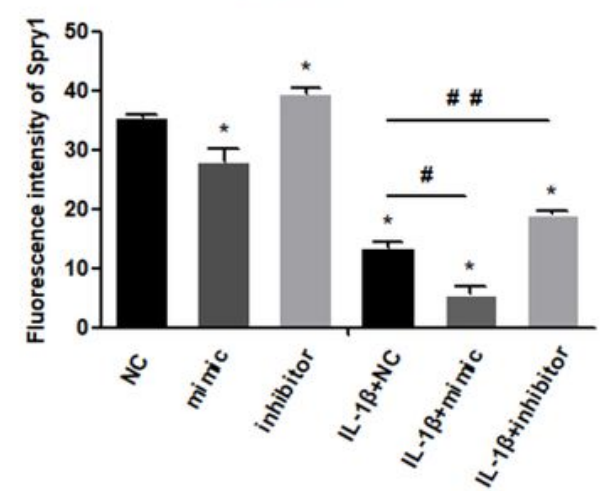

ACAN
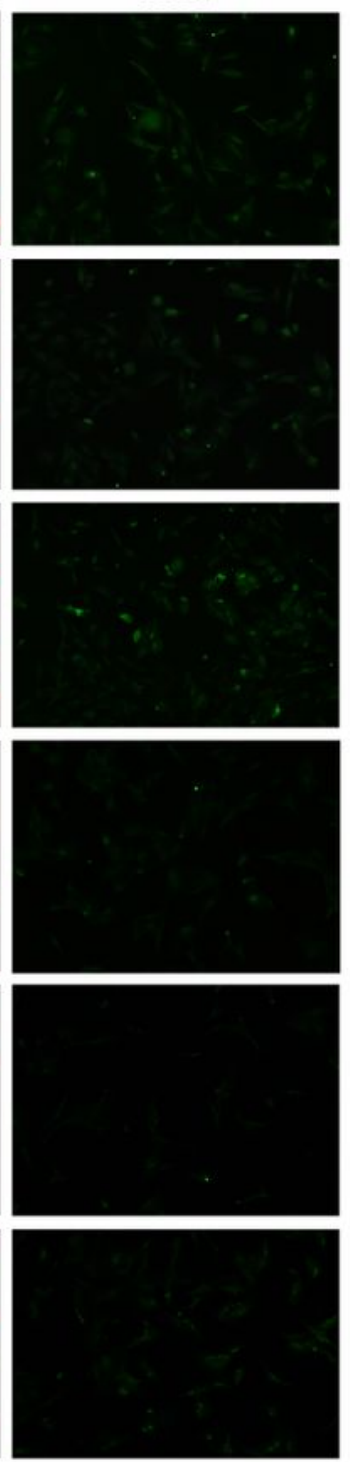

c
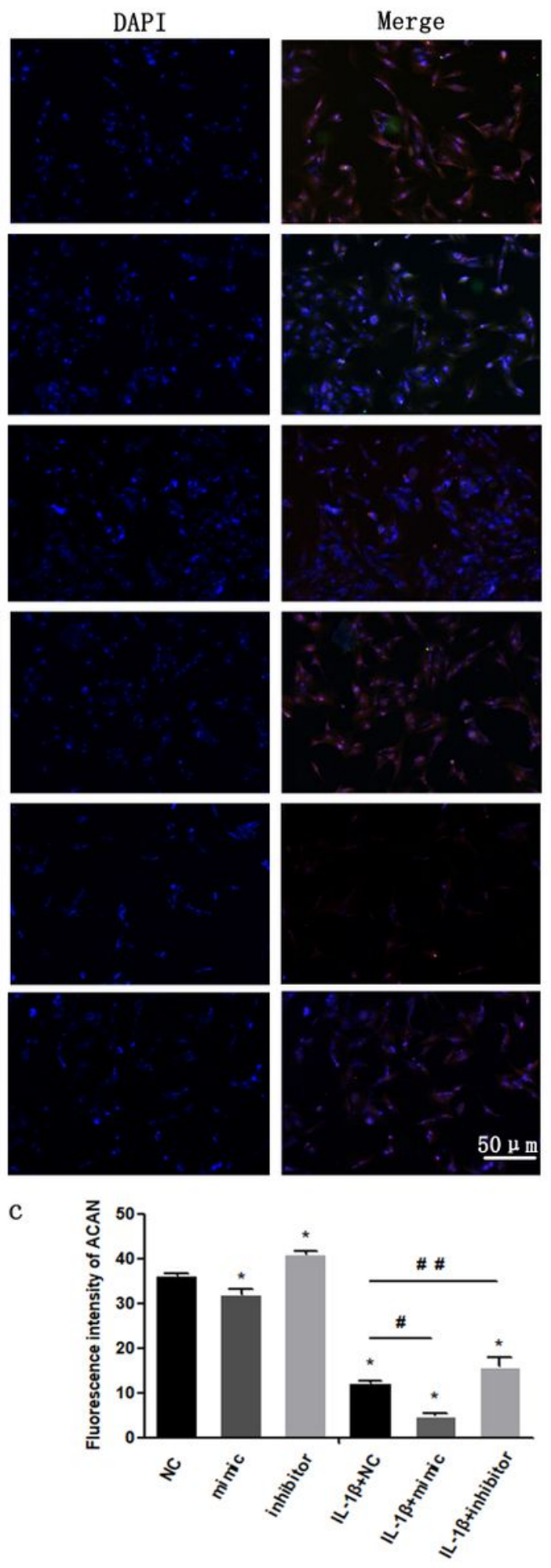

Figure 5 
a Immunofluorescence intensity analysis of Spry 1 and ACAN expression in MCCs. MCCs were transfected with miR-21-5p mimic or miR-21-5p inhibitor and then stimulated with IL-1 $\beta$ for $24 \mathrm{~h}$. Fluorescence micrographs of Spry1 are shown in red (b), ACAN (c) in green, and nuclei in blue. Data are represented as the means \pm standard deviation $(n=3)$. Asterisk $(*)$ : compared with the NC group. Hash (\#\#): compared with the IL-1 $\beta+N C$ group. Data are represented as the means \pm standard deviation $(n=3)$. ${ }^{*}<<0.05, \# P<$ 0.05, \#\#P< 0.05. NS: not significant. Scale bar: $50 \mu \mathrm{m}$.

a

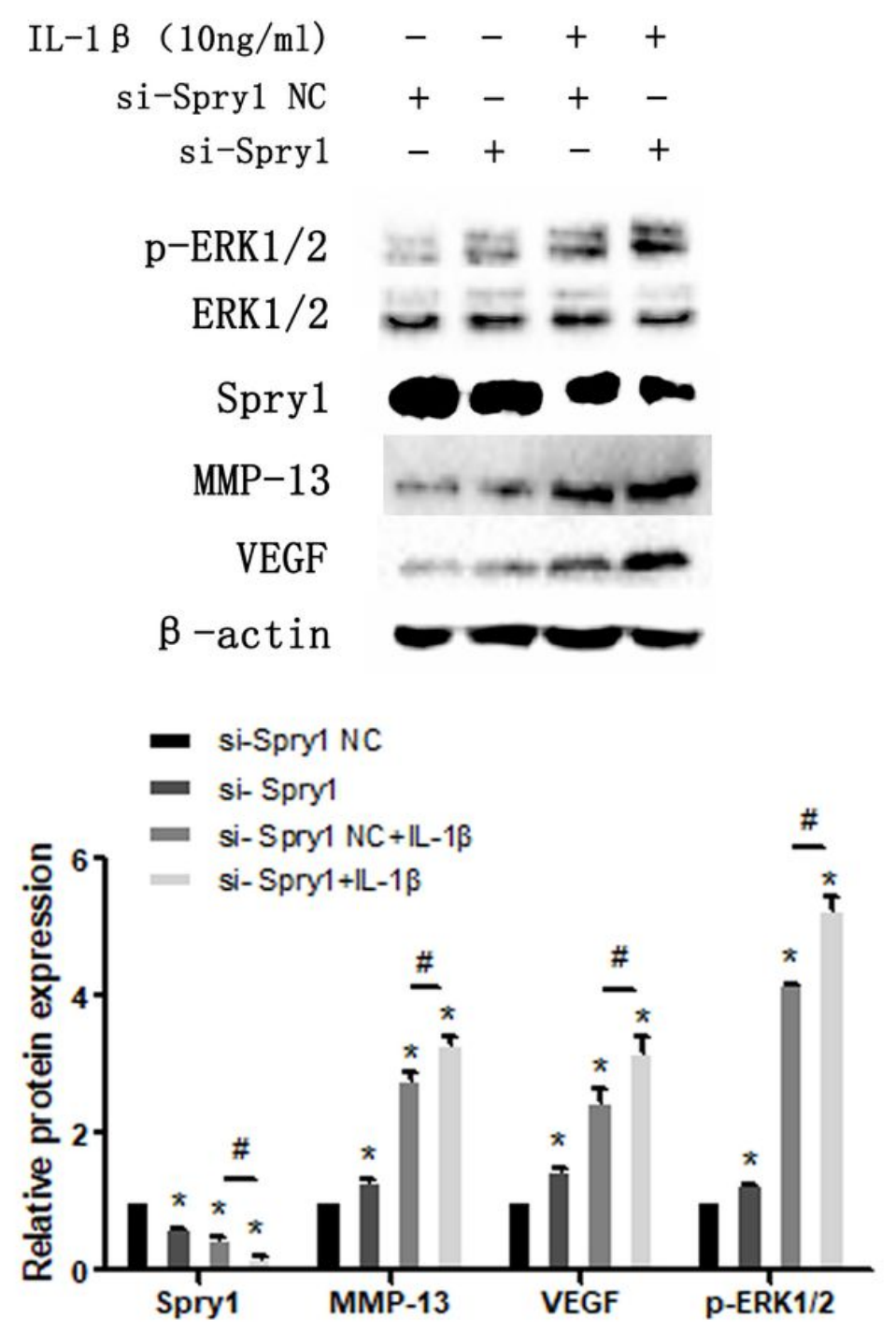

$\mathrm{b}$

$\begin{array}{rllll}\mathrm{IL}-1 \mathrm{\beta}(10 \mathrm{ng} / \mathrm{m} 1) & - & - & + & + \\ \mathrm{cDNANC} & + & - & + & - \\ \mathrm{cDNA} S p r y 1 & - & + & - & +\end{array}$

p-ERK1/2

ERK1/2

Spry1

MMP-13

VEGF

$\beta$-actin
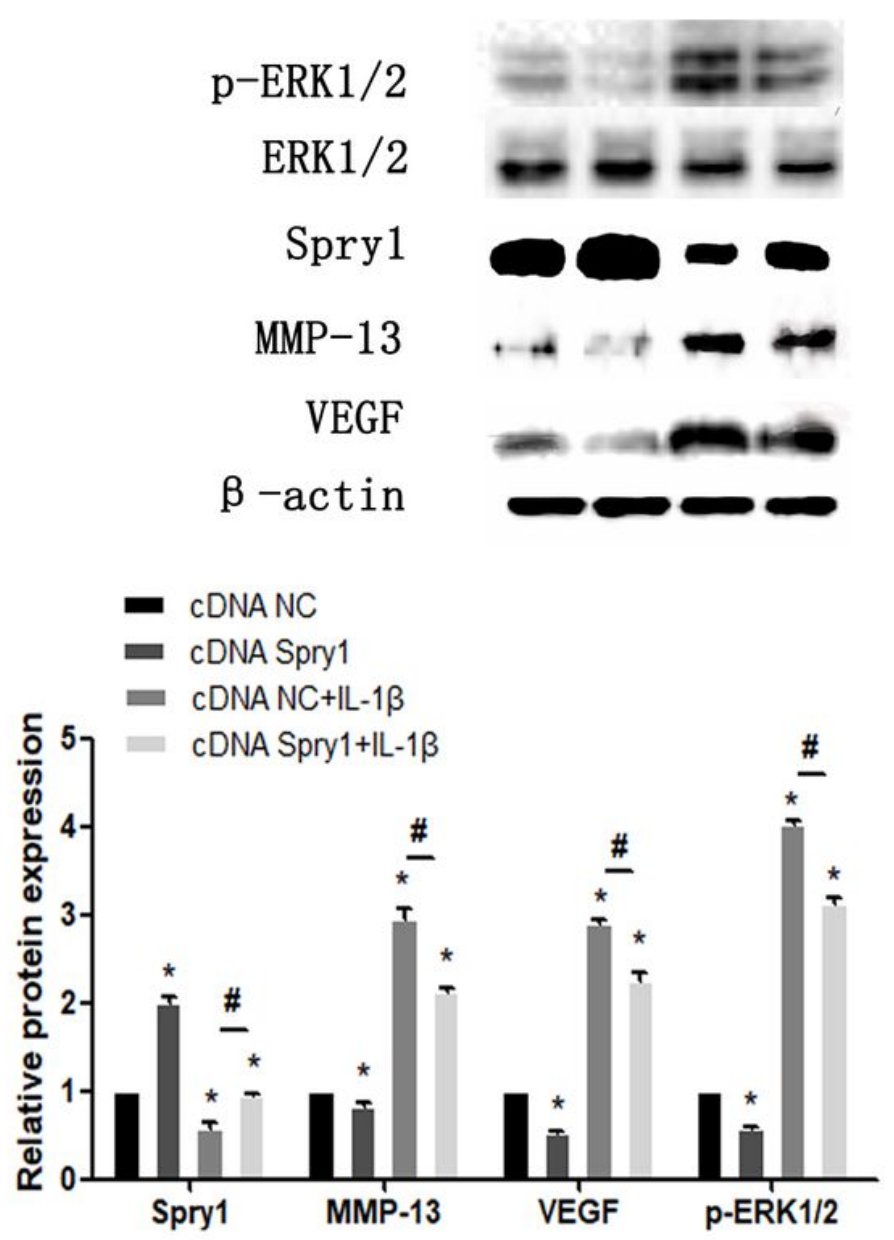

\section{Figure 6}

MiR-21-5p could affect the expression of MCC inflammatory factors by regulating Spry1. a MCCs transfected with Spry1 siRNA (si-Spry 1 ) or negative control (si-NC) were stimulated by $10 \mathrm{ng} / \mathrm{ml}$ IL-1 $\beta$ for $24 \mathrm{~h}$. b MCCs transfected with empty vector (cDNA NC) or Spry1 ectopic expression plasmid (cDNA Spry 1 ) for $24 \mathrm{~h}$ were stimulated by IL-1 $\beta$ for another $24 \mathrm{~h}$. Asterisk (*): compared with the si-NC (a) or cDNA NC (b) group. Hash (\#): compared with the si-NC+IL-1 $\beta$ (a) group or CDNA NC+IL-1 $\beta$ (b) group. Western blot analysis showing the expression of Spry1, MMP-13, VEGF and p-ERK1/2 in MCCs $(a, b)$. Data are represented as the means \pm standard deviation $(n=3)$. ${ }^{*}<0.05, \# P<0.05$. NS: not significant. 
a

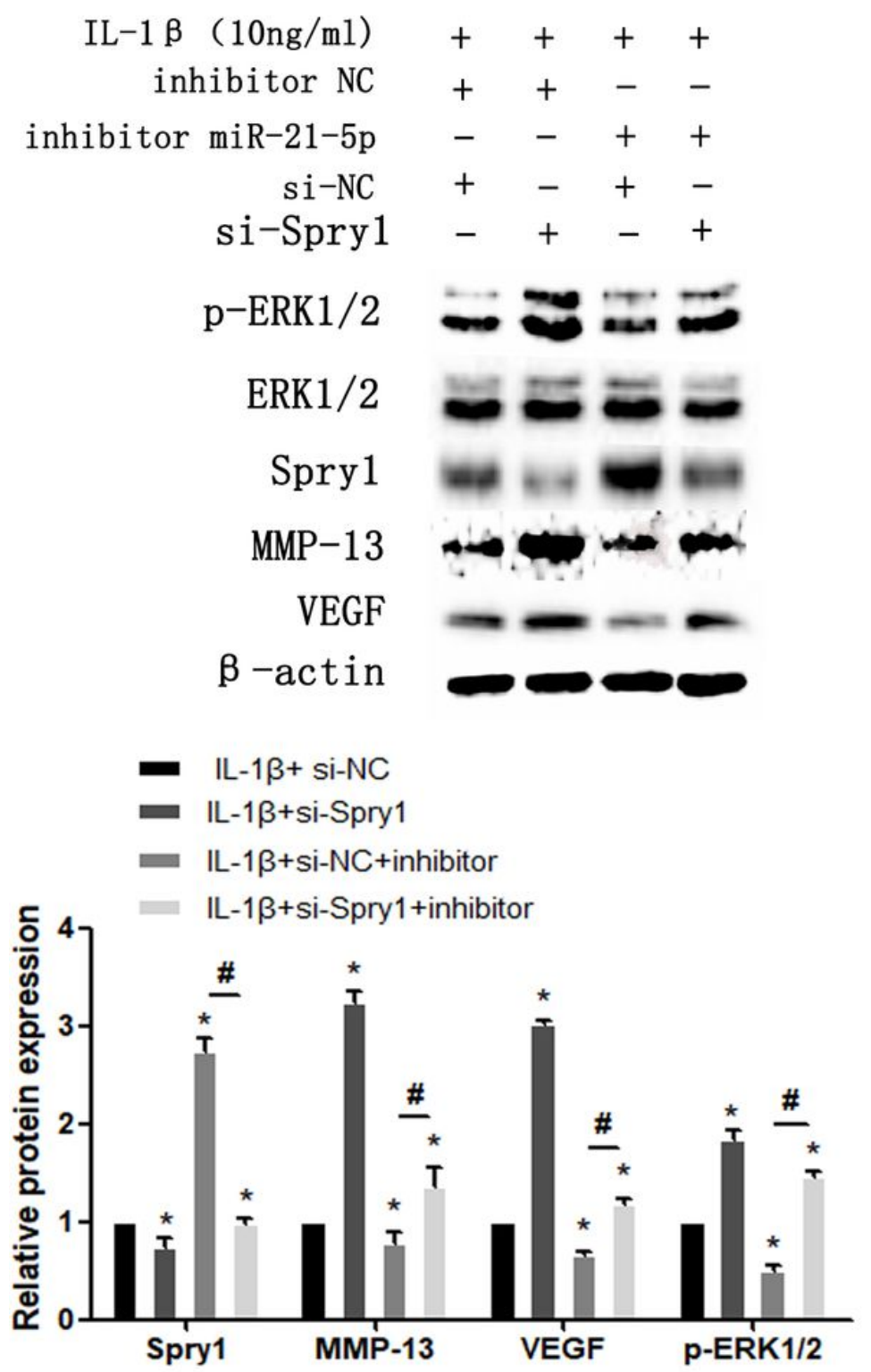

b
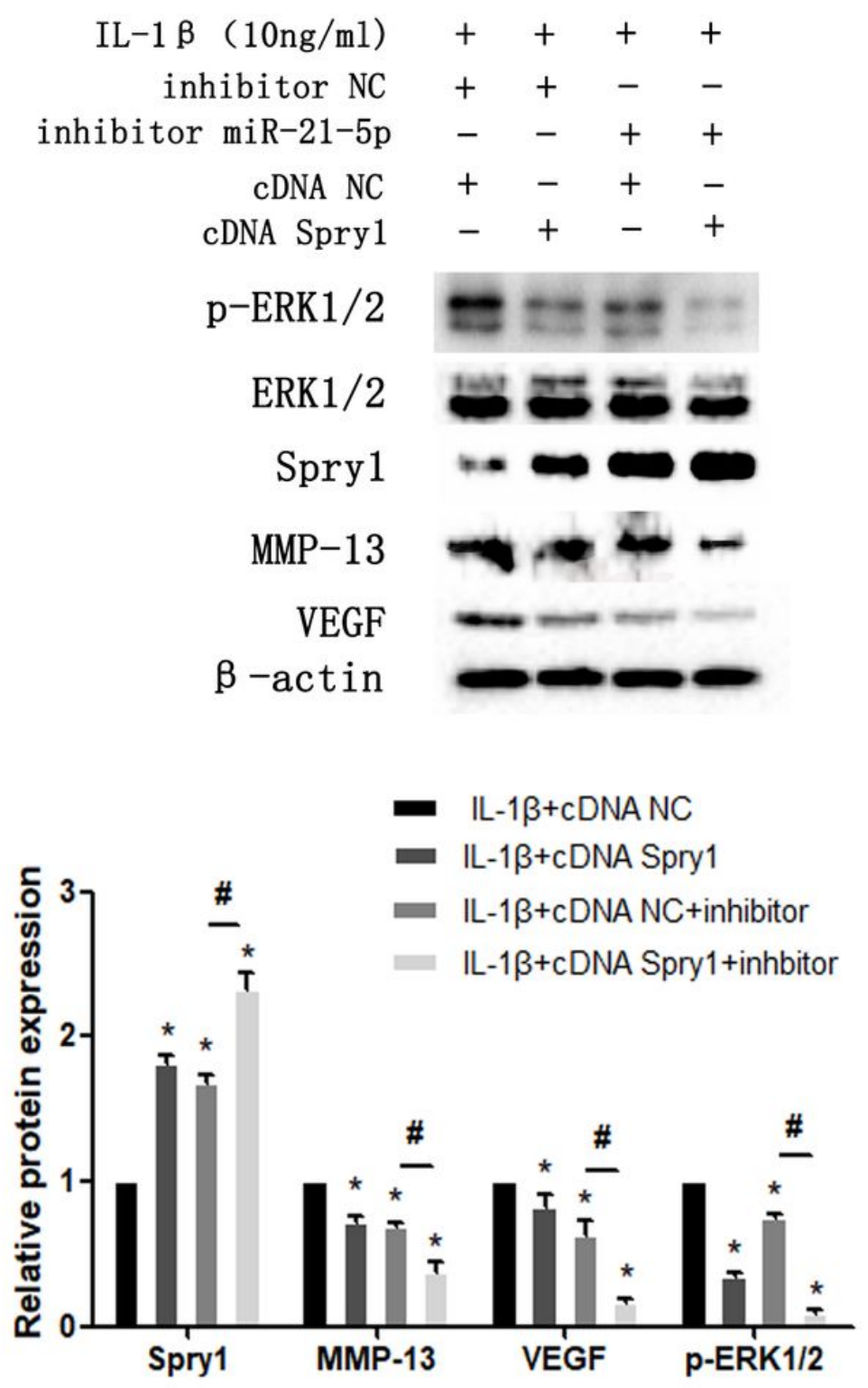

Figure 7

a Under stimulation with IL-1 $\beta$, MCCs were transfected with inhibitor NC or miR-21-5p inhibitor for $24 \mathrm{~h}$ and then treated with Spry 1 siRNA (si-Spry1) for another 24 h. b Under stimulation with IL-1 $\beta$, MCCs were treated with inhibitor NC or miR-21-5p inhibitor for $24 \mathrm{~h}$ and then transfected with the empty vector (cDNA NC) or Spry1 ectopic expression plasmid (cDNA Spry1) for another $24 \mathrm{~h}$. Asterisk (*): compared with the IL-1 $\beta+$ si-NC group (a) or IL-1 $\beta+$ cDNA NC group (b). Hash (\#): compared with the IL-1 $\beta+$ si-NC + inhibitor group (a) or IL-1 $\beta+$ cDNA NC + inhibitor group (b). Western blot analysis showing the expression of Spry 1 , MMP-13, VEGF and p-ERK1/2 in MCCs $(a, b)$. Data are represented as the means \pm standard deviation $(n=$ 3). *P $<0.05, \# P<0.05$. NS: not significant. 
a
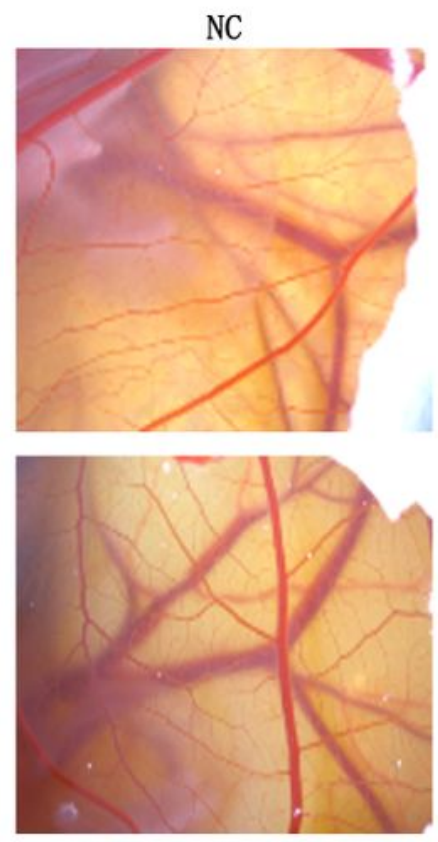

mimic miR-21-5p

$\mathrm{b}$

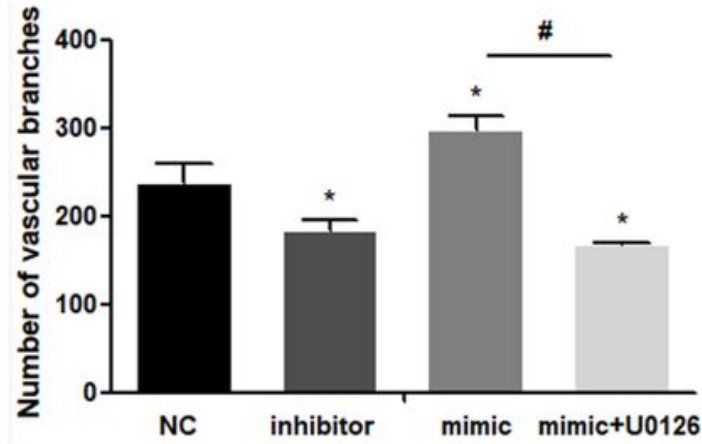

d

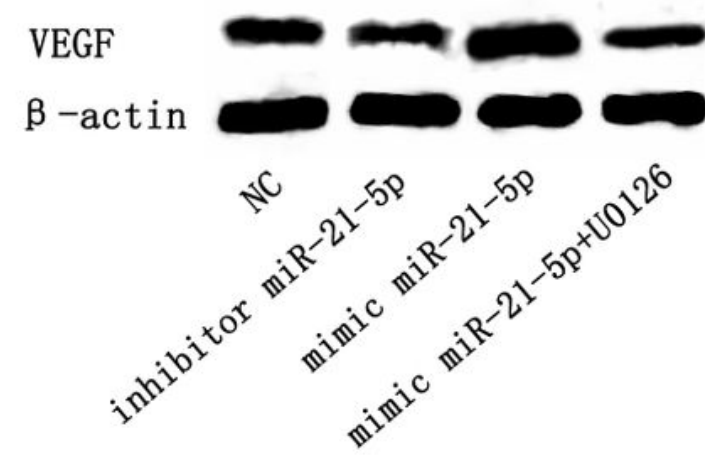

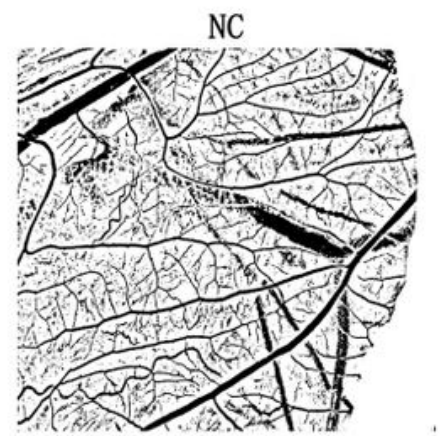
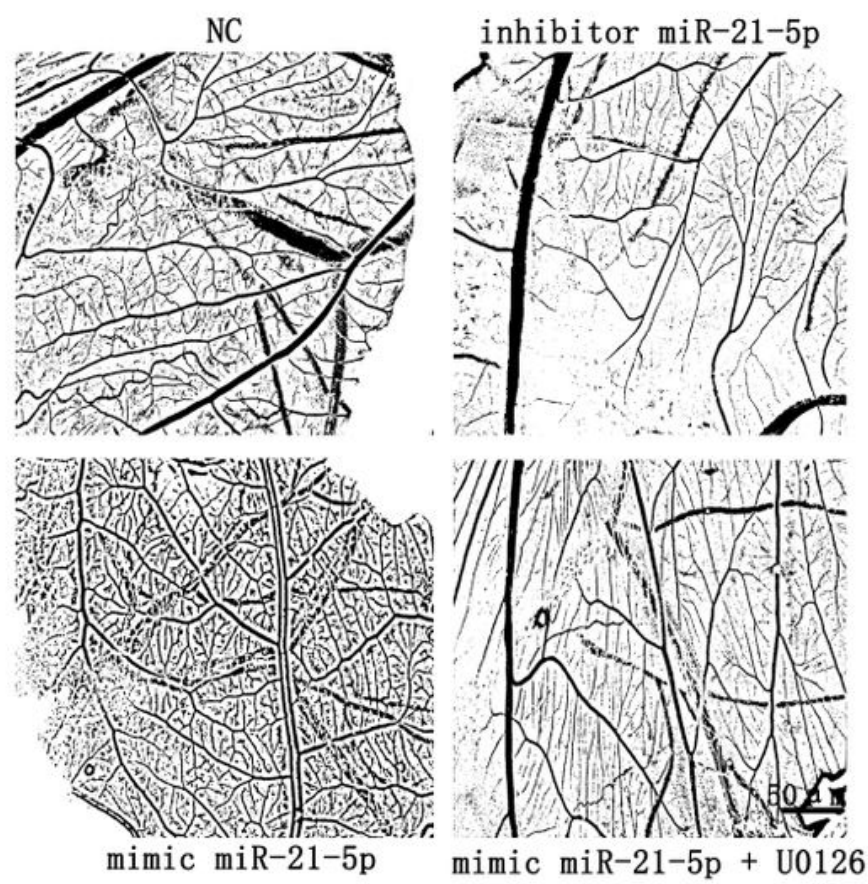

C
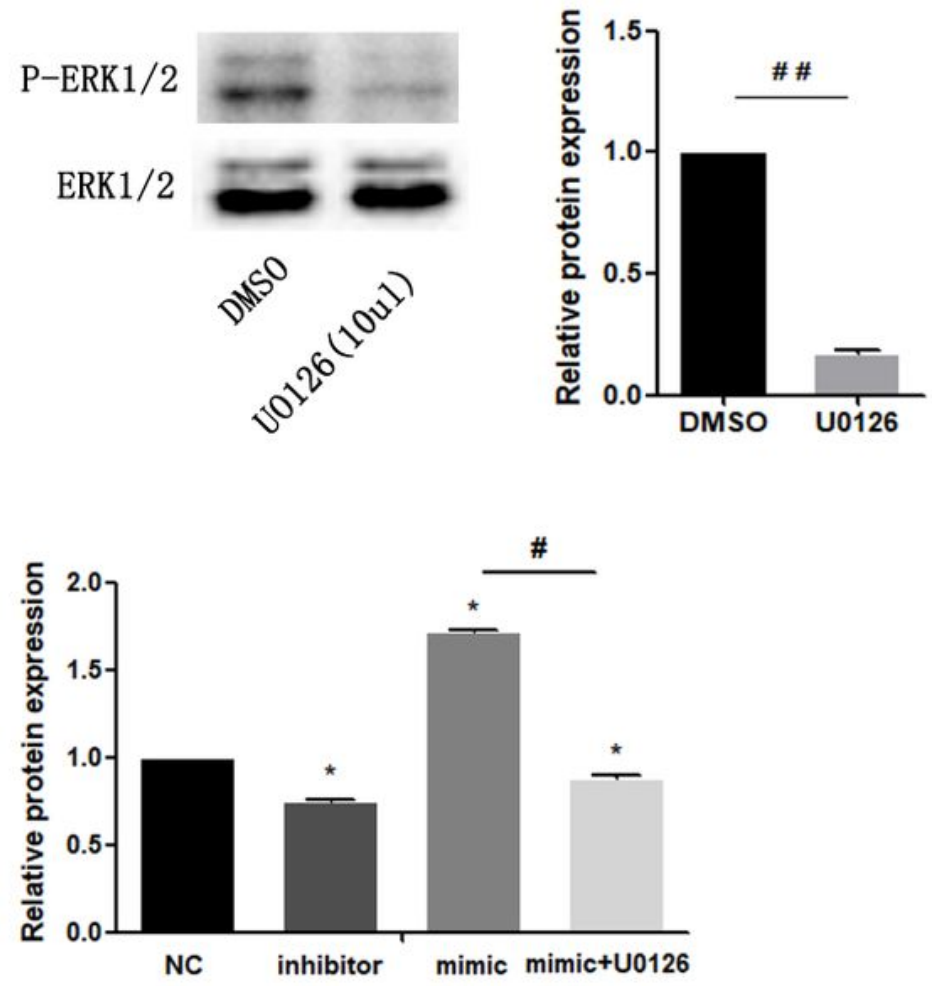

\section{Figure 8}

MiR-21-5p enhances the angiogenesis potential of MCCs by activating the ERK/MAPK signalling pathway. a d MCCs were transfected with miR-21-5p mimic or miR-21-5p inhibitor and treated with U0126. a CAM representative images showing the inoculation area of the different experimental conditions. b Quantitative evaluation of the number of new blood vessels grown towards each inoculation area. MCCs were treated with dimethyl sulfoxide (DMSO) or U0126 (10 $\mu$ l), and changes in the 
expression of p-ERK were determined (c). Detection of VEGF protein expression changes (d). Data are represented as the means \pm standard deviation $(n=8)(b)$. Data are represented as the means \pm standard deviation $(n=3)(c, d)$. Asterisk (*): compared with the negative control (NC) group. Hash (\#): compared with the mimic group. Hash (\#\#): compared with the DMSO group. ${ }^{*} P<0.05, \# P<0.05$, \#\#P<0.05. NS: not significant. Scale bar: $50 \mu \mathrm{m}$.

\section{Supplementary Files}

This is a list of supplementary files associated with this preprint. Click to download.

- Supplementary3.docx

- Supplementary4.docx

- Supplementary1.docx

- Supplementary2.docx 